\title{
Synthesis, Characterization, Antitubercular Activity and Docking Studies of 2-(benzo [d]oxazol-2-ylthio)-1-(3-(4-fluoro-3- methylphenyl)-5-(substituted aryl)-4,5-dihydro-1H-pyrazol-1-yl) ethanone
}

\author{
Muralidharan Venugopal*, Raja Sundararajan, Asha Deepti Choppala \\ Department of Pharmaceutical Chemistry, GITAM Institute of Pharmacy, GITAM (Deemed to be University), Visakhapatnam, Andhra Pradesh-500035, INDIA.
}

\begin{abstract}
A new series of 2-(benzo[d]oxazol-2-ylthio)-1-(3-(4-fluoro-3-methylphenyl)-5(substituted aryl)-4,5-dihydro-1 H-pyrazol-1-yl)ethanone (6a-6j) were designed and synthesized from the intermediate chalcones (1a-1j). The synthesized compounds were characterized by FT-IR, ${ }^{1} \mathrm{H}-\mathrm{NMR}$, Mass spectroscopy and bases of elemental analysis. The agar dilution method (In vitro M. tuberculosis method) was employed for anti-tubercular screening. From the study, it was revealed that compounds $\mathbf{6 b}$ and $\mathbf{6} \mathbf{h}$ showed increased potency. In phenyl ring attached to 4,5-dihydropyrazole ring presence of electron donating substituent like dimethylamino, hydroxy and methoxy moiety mightbe responsible for the powerful anti-mycobacterial activity displayed by derivatives $\mathbf{6} \mathbf{b}$ and $\mathbf{6} \mathbf{h}$. Further docking studies were performed to predict the interactions of the target compounds $\mathbf{6 a}-\mathbf{6 j}$ within the Mycobacterium tuberculosis enoyl reductase enzyme by their scores and mode of binding to amino acids. In addition, drug-likeness score and molecular properties responsible for a good pharmacokinetic profile were calculated by Osiris
\end{abstract}

property explorer and Molinspiration online toolkit, respectively. From the results, it was revealed that the synthesized compounds with electron releasing groups showed the most potent activity compared to that of standard drug.

Key words: Anti-tubercular activity, Benzo[d]oxazole-2-thiol, Chalcones, Docking studies, Pyrazolines.

\section{Correspondence}

Muralidharan V,

Department of Pharmaceutical Chemistry, GITAM Institute of Pharmacy, GITAM (Deemed to be University), Visakhapatnam, Andhra Pradesh-500035, INDIA.

Phone: +919866807070

Email:vmd1213@gmail.com

DOI: 10.5530/jyp.2020.12s.39

\section{INTRODUCTION}

Tuberculosis (TB) is the most powerful communicable bacterial diseases caused by Mycobacterium tuberculosis. ${ }^{1,2}$ It has a good impact on the history of infection on humankind and was called "Captain Among These Men of Death" in $18^{\text {th }}$ and $19^{\text {th }}$ century. It was declared as "Global Public Health Emergency" by WHO., Highest total number of TB cases worldwide in 2010 was reported in India, in part due to poor disease management within the public and private health care sector. Programs such as the Revised National Tuberculosis Control Program are working to reduce TB levels among people receiving public health care. ${ }^{5}$ The increase in the numbers of death cases were based upon the decrease in the efficacy of first four line drugs and increase in resistance to at least isoniazid and rifampicin which was called Multidrug Resistance tuberculosis (MDR-TB).

As a consequence of multiple mutations in specific resistant-associated genes of Mycobacterium tuberculosis (inhA,katG, rpoB, gyrA, rrs, tlyA and eis), extensively drug-resistant (XDR) TB has arisen. ${ }^{6}$ It was shown that treatment of XDR-TB by using isoniazid and rifampicin plus a fluoroquinolone derivative and amikacin, kanamycin or capreomycin is ineffectual. In view of the frequency and emergence of MDR and $\mathrm{XDR}$ tuberculosis and consequences of acquired resistance to clinically employed drugs, researchers have persisted in performing synthesis and anti-tuberculosis evaluation of novel compounds bearing various chemical entities.
Pyrazoline (Dihydropyrazole) is a heterocyclic scaffold of interest to a synthetic chemist, as it has a broad spectrum of pharmacological activity and its ease of synthesis. Pyrazolines possess a sundry of activities including antimicrobial, ${ }^{7}$ anti-diabetic, ${ }^{8}$ antitumor, ${ }^{9}$ antiarrythmic, ${ }^{10}$ anti-inflammatory, ${ }^{11}$ analgesic, ${ }^{12}$ anti-malaria, ${ }^{13}$ anti-oxidant, ${ }^{14}$ CNS activity, ${ }^{15}$ carbonic anhydrase inhibition, ${ }^{16}$ as glycogen synthase kinase (GSK) inhibitors, ${ }^{17}$ cyclin dependent kinase (CDK) inhibitors, ${ }^{18}$ epidermal growth factor receptor (EGFR) inhibitors, ${ }^{19}$ dual src/Ab1 kinase inhibitors. ${ }^{20}$ Considering the findings above and in continuation of our efforts for the development of anti-tubercular agents, we undertook the design and synthesis of some novel prototypes which possess advantage of the two pharmacophores of pyrazolines and benzo[d]oxazole in single molecular backbone

\section{MATERIALS AND METHODS}

The organic solvents such as methanol, ethanol, acetone, chloroform, n-hexane and ethyl acetate were of spectral grade and used as such without further purification. Some of the solvents were purchased from the local distributors of S.D. Fine Chem. Ltd., Mumbai, India.

All the chemicals used in the synthesis were obtained from standard commercial sources. 4-fluoro-3-methyl-acetophenone was purchased from Avra chemicals. Reactions were monitored by TLC using silica gel-G (Merck grade) as the adsorbent and the solvent systems are indicated at appropriate places. Silica gel (100-200 mesh, Merck grade) 
has been used for column chromatography. Each fraction of $100 \mathrm{ml}$ was collected. The separations of the compounds were checked on TLC under UV lamp and also by spraying the plates with $10 \%$ sulphuric acid. All the melting points were determined in open capillaries, using Boitus digital melting point apparatus, expressed in ${ }^{\circ} \mathrm{C}$ and are uncorrected. The ${ }^{1} \mathrm{H}$ NMR spectra of the compounds were recorded on Bruker Ultra Shield $(400 \mathrm{MHz}) \mathrm{NMR}$ spectrometer in $\mathrm{CDCl}_{3}$ using tetramethylsilane $\left[\left(\mathrm{CH}_{3}\right)_{4} \mathrm{Si}\right]$ as the internal standard. Chemical shift $(\delta)$ are expressed in ppm.

The Mass spectra were obtained on a JEOL-SX-102 instrument using electron impact ionization. All the IR spectra were recorded in $\mathrm{KBr}$ pellets on a Jasco FT-IR 410 spectrometer. Elemental analyses were performed on a Perkine Elmer model 240c analyzer and were within $\pm 0.4 \%$ of the theoretical values.

\section{Experimental}

Preparation of (E)-3-(substituted phenyl)-1-(4-fluoro-3methylphenyl)prop-2-en-1-one (3a-3j)

The key intermediates (E)-3-(substituted phenyl)-1-(4-fluoro-3methylphenyl)prop-2-en-1-one (3a-3j) were prepared according to the reported literature. ${ }^{21}$ The starting material 4-fluoro-3-methyl acetophenone $(2.5 \mathrm{mmol})$ was treated with aromatic aldehydes (2.5 mmol) in presence of catalytic amount of lithium hydroxide. Ethanol (20ml) was used as a solvent. The reaction mixture was kept for constant stirring using a multistage magnetic stirrer at room temperature until the solution turns turbid. The reaction was monitored by TLC (n-hexane: acetone - 7:3). Then the reaction mixture was poured into crushed ice and neutralized with the help of dil. $\mathrm{HCl}$. The precipitate was filtered under vacuum, washed with cold ethanol and distilled water. The obtained chalcones were purified by recrystalization and column chromatography.

(E)-1-(4-fluoro-3-methylphenyl)-3-(thiophen-2-yl)prop-2-en-1-one (3a) Greenish yellow crystals $\left(\right.$ EtOH), Yield $=81 \%$; mp 101-103 ${ }^{\circ} \mathrm{C}$. FT-IR $(\mathrm{KBr}) \mathrm{cm}^{-1}$ : 1657 (C=O, Chalcone), 1585 (C=C), 1149 (C-F), 2949 $\left(\mathrm{C}-\mathrm{CH}_{3}\right),{ }^{1} \mathrm{HNMR}\left(400 \mathrm{MHz}, \mathrm{CDCl}_{3} \delta \mathrm{ppm}\right): 2.358\left(\mathrm{~s}, 3 \mathrm{H}, \mathrm{CH}_{3}\right), 7.430$ (d, 1H, $\alpha-\mathrm{H}), 7.921(\mathrm{~d}, 1 \mathrm{H}, \beta-\mathrm{H}), 7.42(\mathrm{~s}, 1 \mathrm{H}, \mathrm{Ar}-\mathrm{H}), 7.32(\mathrm{~d}, 1 \mathrm{H}, \mathrm{Ar}-\mathrm{H})$, 7.848 (d, 1H, Ar-H), 7.86 (d, 1H, Ar-H), 7.433 (d, 1H, Ar-H), 7.972 (s, 1H, Ar-H). MS (EI) m/z: $247\left(\mathrm{M}^{+}\right)$. Anal. Calcd for $\mathrm{C}_{14} \mathrm{H}_{11}$ FOS: C, 68.27; H, 4.50; F, 7.71; O, 6.50; S, 13.02 .

(E)-1-(4-fluoro-3-methylphenyl)-3-(3,4,5-trimethoxyphenyl)prop-2-en1-one (3b)

Lemon yellow crystals $(\mathrm{EtOH})$, Yield $=86 \%$; mp 95-97 C. FT-IR $(\mathrm{KBr})$ $\mathrm{cm}^{-1}: 1651$ (C=O, Chalcone), 1585(C=C), $1244\left(\mathrm{C}-\mathrm{O}-\mathrm{CH}_{3}\right), 2924$ $\left(\mathrm{C}-\mathrm{CH}_{3}\right), 1122(\mathrm{C}-\mathrm{F}) .{ }^{1} \mathrm{HNMR}\left(400 \mathrm{MHz}, \mathrm{CDCl}_{3} \delta \mathrm{ppm}\right): 2.38(\mathrm{~s}, 3 \mathrm{H}$, $\left.-\mathrm{CH}_{3}\right), 3.952\left(\mathrm{~d}, 9 \mathrm{H},-\mathrm{OCH}_{3}\right), 7.758(\mathrm{~d}, 1 \mathrm{H}, \alpha-\mathrm{H}), 7.926(\mathrm{~d}, 1 \mathrm{H}, \beta-\mathrm{H})$, 7.719 (s, 1H, Ar-H), 7.14(d, 1H, Ar-H), 6.889 (d, 1H, Ar-H), 7.12(s, 1H, Ar-H), 7.28(s, 1H, Ar-H). MS (EI) m/z: $331\left(\mathrm{M}^{+}\right)$. Anal. Calcd for $\mathrm{C}_{19} \mathrm{H}_{19} \mathrm{FO}_{4}: \mathrm{C}, 69.08 ; \mathrm{H}, 80 ; \mathrm{F}, 5.75 ; \mathrm{O}, 19.37$.

(E)-3-(2-bromophenyl)-1-(4-fluoro-3-methylphenyl) prop-2-en-1-one (3c) Yellow crystals $(\mathrm{EtOH})$, Yield $=76 \%$; mp $100-102^{\circ} \mathrm{C}$. FT-IR $(\mathrm{KBr}) \mathrm{cm}^{-1}$ :

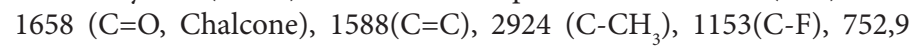
(C-Br). ${ }^{1} \mathrm{HNMR}\left(400 \mathrm{MHz}, \mathrm{CDCl}_{3} \delta \mathrm{ppm}\right): 2.384\left(\mathrm{~s}, 3 \mathrm{H},-\mathrm{CH}_{3}\right), 7.374$ (d, 1H, $\alpha-\mathrm{H}), 8.155$ (d, 1H, $\beta-\mathrm{H}), 7.119-7.93$ (d, 7H, Ar-H). MS (EI) m/z: $319\left(\mathrm{M}^{+}\right)$. Anal. Calcd for $\mathrm{C}_{16} \mathrm{H}_{12}$ BrFO: C, 60.21; H, 3.79; F, 5.95; O, 5.01; $\mathrm{Br}, 25.04$.

(E)-1-(4-fluoro-3-methylphenyl)-3-(2-nitrophenyl)prop-2-en-1-on (3d)

Orange crystals $(\mathrm{EtOH})$, Yield $=77 \%$; $\mathrm{mp} 94-96^{\circ} \mathrm{C}$. FT-IR $(\mathrm{KBr}) \mathrm{cm}^{-1}$ : $1674\left(\mathrm{C}=\mathrm{O}\right.$, Chalcone), 1513(C=C), $2878\left(\mathrm{C}-\mathrm{CH}_{3}\right), 1292(\mathrm{C}-\mathrm{F}), 1341$
$\left(\mathrm{C}-\mathrm{NO}_{2}\right.$ ). ${ }^{1} \mathrm{HNMR}\left(400 \mathrm{MHz}, \mathrm{CDCl}_{3} \delta \mathrm{ppm}\right): 2.39$ (s, 3H, $\left.\mathrm{CH}_{3}\right), 7.86$ (d, $1 \mathrm{H}, \alpha-\mathrm{H}), 8.09$ (d, $1 \mathrm{H}, \beta-\mathrm{H}), 7.585$ (s, 1H, Ar-H), 7.173 (d, 1H, Ar-H), $7.71(\mathrm{~d}, 1 \mathrm{H}, \mathrm{Ar}-\mathrm{H}) 8.15$ (s, 1H, Ar-H), $8.112(\mathrm{~d}, 1 \mathrm{H}, \mathrm{Ar}-\mathrm{H}), 7.582(\mathrm{~d}, 1 \mathrm{H}$, Ar-H), 7.928 (d, Ar-H). MS (EI) m/z: $284\left(\mathrm{M}^{+}\right)$. Anal. Calcd for $\mathrm{C}_{16} \mathrm{H}_{12} \mathrm{FNO}_{3}$ : C, 67.36; H, 4.24; F, 6.66; N, 4.91;O,16.83.

(E)-3-(4-bromophenyl)-1-(4-fluoro-3-methylphenyl) prop-2-en-1-one (3e) Yellow crystals $(\mathrm{EtOH})$, Yield $=76 \%$; mp $100-102^{\circ} \mathrm{C}$. FT-IR $(\mathrm{KBr}) \mathrm{cm}^{-1}$ : 1645 (C=O, Chalcone), 1593(C=C), $2941\left(\mathrm{C}-\mathrm{CH}_{3}\right), 1103(\mathrm{C}-\mathrm{F}), 759$ (C-Br). ${ }^{1} \mathrm{HNMR}\left(400 \mathrm{MHz}, \mathrm{CDCl}_{3} \delta \mathrm{ppm}\right): 2.371\left(\mathrm{~s}, 3 \mathrm{H},-\mathrm{CH}_{3}\right), 7.342$ (d, 1H, a-H), 8.015 (d, 1H, $\beta-\mathrm{H}), 7.109-7.92$ (d, 7H, Ar-H). MS (EI) m/z: $319\left(\mathrm{M}^{+}\right)$. Anal. Calcd for $\mathrm{C}_{16} \mathrm{H}_{12}$ BrFO: C, 60.21; H, 3.79; F, 5.95; O, 5.01; $\mathrm{Br}, 25.04$.

(E)-3-(3,4-dimethoxyphenyl)-1-(4-fluoro-3-methylphenyl) prop-2-en-1one (3f)

Lemon yellow crystals $(\mathrm{EtOH})$, Yield $=81 \%$; mp $85-87^{\circ} \mathrm{C}$. FT-IR $(\mathrm{KBr})$ $\mathrm{cm}^{-1}: 1656$ (C=O, Chalcone), 1583(C=C), $2930\left(\mathrm{C}-\mathrm{CH}_{3}\right), 1254(\mathrm{C}-\mathrm{F})$, $1142\left(\mathrm{C}-\mathrm{OCH}_{3}\right) .{ }^{1} \mathrm{HNMR}\left(400 \mathrm{MHz}, \mathrm{CDCl}_{3} \delta \mathrm{ppm}\right): 2.35$ (s, 3H, $\left.\mathrm{CH}_{3}\right)$, $3.936\left(\mathrm{~d}, 6 \mathrm{H},-\mathrm{OCH}_{3}\right), 7.739(\mathrm{~d}, 1 \mathrm{H}, \alpha-\mathrm{H}), 7.905(\mathrm{~d}, 1 \mathrm{H}, \beta-\mathrm{H}), 7.38(\mathrm{~s}, 1 \mathrm{H}$, Ar-H), 7.25 (d, 1H, Ar-H), 7.864 (d, 1H, Ar-H), 7.23 (s, 1H, Ar-H), 7.080 (d, 1H, Ar-H), 6.913 (d, 1H, Ar-H). MS (EI) m/z: $301\left(\mathrm{M}^{+}\right)$. Anal. Calcd for $\mathrm{C}_{18} \mathrm{H}_{17} \mathrm{FO}_{3}$ : C, 71.99; $\mathrm{H}, 5.71 ; \mathrm{F}, 6.33 ; \mathrm{O}, 15.98$.

(E)-3-(4-chlorophenyl)-1-(4-fluoro-3-methylphenyl) prop-2-en-1-one (3g)

Lemon yellow crystals $(\mathrm{EtOH})$, Yield $=77 \% ; \mathrm{mp} 101-103^{\circ} \mathrm{C}$. FT-IR $(\mathrm{KBr})$ $\mathrm{cm}^{-1}: 1663$ (C=O, Chalcone), 1591(C=C), $2960\left(\mathrm{C}-\mathrm{CH}_{3}\right), 1243(\mathrm{C}-\mathrm{F}), 819$ $(\mathrm{C}-\mathrm{Cl}) .{ }^{1} \mathrm{HNMR}\left(400 \mathrm{MHz}, \mathrm{CDCl}_{3} \delta \mathrm{ppm}\right): 2,38$ (s, $\left.3 \mathrm{H}, \mathrm{CH}_{3}\right), 7,591$ (d, 1H, $\alpha-\mathrm{H}), 7,93$ (d, 1H, $\beta-\mathrm{H}), 7,58$ (s, 1H, Ar-H), 7,433 (d, 1H, Ar-H), 7,61 (d, 1H, Ar-H), 7,60 (s, 1H, Ar-H), 7,75 (d, 1H, Ar-H), 7,47 (d, 1H, Ar-H). MS (EI) m/z: $275\left(\mathrm{M}^{+}\right)$. Anal. Calcd for $\mathrm{C}_{16} \mathrm{H}_{12}$ ClFO: C, 69.95; H, 4.40; Cl, 12.91; F, 6.92; O, 5.82.

(E)-3-(4-(dimethylamino)phenyl)-1-(4-fluoro-3-methylphenyl)prop-2en-1-one (3h)

Bright red crystals $(\mathrm{EtOH})$, Yield $=83 \% ; \mathrm{mp} 93-95^{\circ} \mathrm{C} . \mathrm{FT}-\mathrm{IR}(\mathrm{KBr}) \mathrm{cm}^{-1}: 1651$

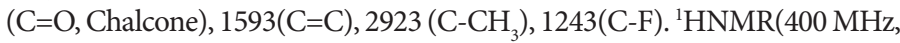
$\mathrm{CDCl}_{3} \delta \mathrm{ppm}$ ): $2.37\left(\mathrm{~s}, 3 \mathrm{H}, \mathrm{CH}_{3}\right), 3.06\left(\mathrm{~s}, 6 \mathrm{H}, \mathrm{N}\left(\mathrm{CH}_{3}\right), 7.913(\mathrm{~d}, 1 \mathrm{H}\right.$, $\alpha-\mathrm{H}), 7.577$ (d, 1H, $\beta-\mathrm{H}), 7.34$ (s, 1H, Ar-H), 7.03 (d, 1H, Ar-H), 7.849 (d, 1H, Ar-H), 7.78 (s, 2H, Ar-H), 6.734 (d, 2H, Ar-H). MS (EI) m/z: 284 $\left(\mathrm{M}^{+}\right)$. Anal. Calcd for $\mathrm{C}_{18} \mathrm{H}_{18}$ FNO: C, 76.30; H, 6.40; F, 6.71; O, 5.65; N, 4.94 .

(E)-1-(4-fluoro-3-methylphenyl)-3-(4-hydroxy-3-methoxy-5-nitrophenyl) prop-2-en-1-one. (3i)

Pale yellow crystals $\left(\right.$ EtOH), Yield $=78 \%$; $\mathrm{mp} 91-93^{\circ} \mathrm{C}$. FT-IR $(\mathrm{KBr}) \mathrm{cm}^{-1}$ : 1684 (C=O, Chalcone), 1546(C=C), $2944\left(\mathrm{C}-\mathrm{CH}_{3}\right), 1103(\mathrm{C}-\mathrm{F}), 1230$ $\left(\mathrm{C}-\mathrm{OCH}_{3}\right), 1366\left(\mathrm{NO}_{2}\right), 3200(\mathrm{OH}) .{ }^{1} \mathrm{HNMR}\left(400 \mathrm{MHz}, \mathrm{CDCl}_{3} \delta \mathrm{ppm}\right)$ $\mathrm{CH}_{3}: 2.186$ (s, 3H, $\left.\mathrm{CH}_{3}\right), 1.727(\mathrm{~s}, 1 \mathrm{H}, \mathrm{OH}) 4.043\left(\mathrm{~s}, 3 \mathrm{H},-\mathrm{OCH}_{3}\right), 7.664$ (d, 1H, $\alpha-\mathrm{H}), 8.245$ (d, 1H, $\beta-\mathrm{H}), 7.285-8.245(\mathrm{~m}, 5 \mathrm{H}, \mathrm{Ar}-\mathrm{H})$. MS (EI) $\mathrm{m} / \mathrm{z}: 331,09\left(\mathrm{M}^{+}\right)$. Anal. Calcd for $\mathrm{C}_{17} \mathrm{H}_{14} \mathrm{NO}_{5}$ : C, 61.63; H, 4.26; F, 5.73; $\mathrm{N}, 4.23$; O, 24.15.

(E)-3-(5-bromo-2-hydroxy-3-methoxyphenyl)-1-(4-fluoro-3methylphenyl)prop-2-en-1-one (3j)

Orange crystals $(\mathrm{EtOH})$, Yield $=69 \% ; \mathrm{mp} 93-95^{\circ} \mathrm{C}$, FT-IR $(\mathrm{KBr}) \mathrm{cm}^{-1}$ :

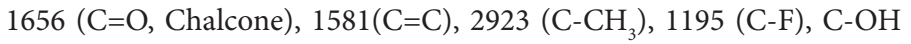
(3243), $\mathrm{C}-\mathrm{OCH}_{3}$ (1255), C-Br (705): ${ }^{1} \mathrm{HNMR}\left(400 \mathrm{MHz}, \mathrm{CDCl}_{3,} \delta \mathrm{ppm}\right)$ : 1,617 (s, 3H, $\mathrm{CH}_{3}$ ), 7,34 (d, 1H, $\left.\alpha-\mathrm{H}\right), 9,879$ (s, $\left.1 \mathrm{H}, \beta-\mathrm{H}\right), 7,203$ (d, $5 \mathrm{H}$, Ar-H), 7,285(s, 1H, OH), 3,943(s, 3H, C-OCH ${ }_{3}$ : MS (EI) m/z: $365\left(\mathrm{M}^{+}\right)$: Anal. Calcd for $\mathrm{C}_{17} \mathrm{H}_{14} \mathrm{FBrO}_{3}$ : C, 55.91; H, 3.83; Br, 21.88; F, 5.20; O, 13.14 . 
Venugopal, et al.: 2-Benzo [d] oxazol-2ylthio Linked Pyrazolines as Potent Anti-TB Agents

Preparation of 3-(3-chloro-4-fluorophenyl)-5-(substituted phenyl)-4,5dihydro-1H-pyrazole (4a-4j)

The previously synthesized (E)-3-(substituted phenyl)-1-(4-fluoro-3methyl phenyl) prop-2-en-1-one $(2.5 \mathrm{mmol})$ was refluxed with hydrazine hydride (5mmol) upto 6 to $8 \mathrm{~h}$ in presence of $20 \mathrm{ml}$ of glacial acetic acid. ${ }^{22}$ Further, the reaction was monitored by TLC and the solvent was evaporated in vacuum. The crushed ice was added to the residue while mixing thoroughly after completion of the reaction. The precipitate formed was filtered under vacuum. The crude products were recrystallized from a suitable solvent to yield the analytically pure product.

3-(4-fluoro-3-methylphenyl)-5-(thiophen-2-yl)-4,5-dihydro-1H-pyrazole. (4a)

Dark greenish crystals $(\mathrm{MeOH})$, Yield $=70 \% ; \mathrm{mp} 94-96^{\circ} \mathrm{C}$. FT-IR $(\mathrm{KBr})$ $\mathrm{cm}^{-1}: 1008$ (C-F), 2941 (C-CH 3 Str), $1590(\mathrm{C}=\mathrm{N}), 1463$ ( $\mathrm{CH}_{2}$ bend), 3657 (Ar-CH). ${ }^{1} \mathrm{HNMR}\left(400 \mathrm{MHz}, \mathrm{CDCl}_{3} \delta \mathrm{ppm}\right) 2.332\left(\mathrm{~s}, 3 \mathrm{H}, \mathrm{CH}_{3}\right), 1.213-$ $1.304\left(\mathrm{~d}, 2 \mathrm{H}, \mathrm{C}_{4}-\mathrm{H}\right.$ Pyrazoline), $3.491\left(\mathrm{t}, 1 \mathrm{H}, \mathrm{C}_{5}-\mathrm{H}\right.$ pyrazoline), 4.928 (d, 1H, NH), 6.87 - 7.38 (6H, Ar-H). MS (EI) m/z: $260\left(\mathrm{M}^{+}\right)$: Anal. Calcd for $\mathrm{C}_{14} \mathrm{H}_{13} \mathrm{FN}_{2} \mathrm{~S}$ : C, 71.40; H, 5.09; N, 8.33. Found: C, 71.25; H, 4.97; $\mathrm{N}, 8.24$.

\section{3-(4-fluoro-3-methylphenyl)-5-(3,4,5-trimethoxyphenyl)-4,5-dihydro- $1 \mathrm{H}$-pyrazole (4b)}

Reddish crystals $(\mathrm{MeOH})$, Yield $=62 \% ; \mathrm{mp} 102-104^{\circ} \mathrm{C}$. FT-IR $(\mathrm{KBr})$ $\mathrm{cm}^{-1}$ : 992 (C-F), 2924 (C-CH ${ }_{3}$ Str), $1561(\mathrm{C}=\mathrm{N}), 1468$ ( $\mathrm{CH}_{2}$ bend), 3481

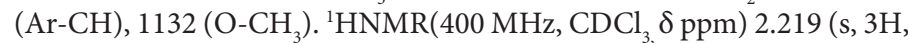
$\left.\mathrm{CH}_{3}\right), 1.246-1.251$ (d, 2H, C $-\mathrm{H}$ Pyrazoline), 4.081 (t, 1H, C $-\mathrm{H}$ Pyrazoline), $3.813,3.663,3.522\left(\mathrm{~s}, 9 \mathrm{H}, \mathrm{O}-\mathrm{CH}_{3}\right), 5.126(\mathrm{~d}, 1 \mathrm{H}, \mathrm{NH}), 6.75-7.72$ $(5 \mathrm{H}, \mathrm{Ar}-\mathrm{H})$. MS (EI) m/z: $344\left(\mathrm{M}^{+}\right)$. Anal. Calcd for $\mathrm{C}_{19} \mathrm{H}_{21} \mathrm{FN}_{2} \mathrm{O}_{3}$ : C, 66.27; H, 6.15; N, 8.13. Found: C, 65.86; H, 6.07; N, 8.04.

\section{5-(2-bromophenyl)-3-(4-fluoro-3-methylphenyl)-4,5-dihydro-1H- pyrazole (4c)}

Reddish brown colour crystals $(\mathrm{MeOH})$, Yield $=64 \%$; $\mathrm{mp} 98-100^{\circ} \mathrm{C}$. FT-IR (KBr) cm ${ }^{-1}: 1013$ (C-F), $2944\left(\mathrm{C}-\mathrm{CH}_{3} \mathrm{Str}\right), 1564(\mathrm{C}=\mathrm{N}), 1355\left(\mathrm{CH}_{2}\right.$ bend), 3425 (Ar-CH), 771 (C-Br). ${ }^{1} \mathrm{HNMR}\left(400 \mathrm{MHz}, \mathrm{CDCl}_{3} \delta \mathrm{ppm}\right)$ 2.311 (s, 3H, $\mathrm{CH}_{3}$ ), 1.610-1.241 (d, 2H, C $-\mathrm{H}$ Pyrazoline), 3.911-3.938 (t, 1H, C $-\mathrm{H}$ Pyrazoline), 4.843 (d, 1H, NH), 7.08-7.68 (7H, Ar-H). MS (EI) $\mathrm{m} / \mathrm{z}: 332\left(\mathrm{M}^{+}\right)$. Anal. Calcd for $\mathrm{C}_{16} \mathrm{H}_{14} \mathrm{BrFN}_{2}$ : C, 57.68; H, 4.24; $\mathrm{N}, 8.41$. Found: C, 57.62; H, 4.01; N, 8.31

\section{3-(4-fluoro-3-methylphenyl)-5-(2-nitrophenyl)-4,5-dihydro-1H-pyrazole} (4d)

Red crystals $(\mathrm{MeOH})$, Yield $=70 \%$; mp $89-91^{\circ} \mathrm{C}$. FT-IR $(\mathrm{KBr}) \mathrm{cm}^{-1}$ : 1081 (C-F), 2926 (C-CH 3 Str), $1574(\mathrm{C}=\mathrm{N}), 1352$ ( $\mathrm{CH}_{2}$ bend), 3309 (Ar-CH), 1527 and $1495\left(\mathrm{C}-\mathrm{NO}_{2}, \mathrm{Str}\right) .{ }^{1} \mathrm{HNMR}\left(400 \mathrm{MHz}, \mathrm{CDCl}_{3} \delta \mathrm{ppm}\right) 2.335$ (s, 3H, $\mathrm{CH}_{3}$ ), 1.625 (d, 2H, C $-\mathrm{H}$ Pyrazoline), 4.059 (t, 1H, $\mathrm{C}_{5}-\mathrm{H}$ Pyrazoline), 5.213 (d, 1H, NH), 6.92-7.72 (7H, Ar-H). MS (EI) m/z: 299 $\left(\mathrm{M}^{+}\right)$. Anal. Calcd for $\mathrm{C}_{16} \mathrm{H}_{14} \mathrm{FN}_{3} \mathrm{O}_{2}: \mathrm{C}, 64.21 ; \mathrm{H}, 4.71 ; \mathrm{N}, 14.04$. Found: C, $64.18 ; \mathrm{H}, 4.64 ; \mathrm{N}, 14.01$

\section{5-(4-bromophenyl)-3-(4-fluoro-3-methylphenyl)-4,5-dihydro-1H- pyrazole (4e)}

Maroon colour crystals $(\mathrm{MeOH})$, Yield $=68 \%$; mp $104-106^{\circ} \mathrm{C}$. FT-IR $(\mathrm{KBr}) \mathrm{cm}^{-1}: 1171$ (C-F), $2941\left(\mathrm{C}-\mathrm{CH}_{3} \mathrm{Str}\right), 1583(\mathrm{C}=\mathrm{N}), 1391\left(\mathrm{CH}_{2}\right.$ bend), 3321 (Ar-CH), 776 (C-Br). ${ }^{1} \mathrm{HNMR}\left(400 \mathrm{MHz}, \mathrm{CDCl}_{3} \delta \mathrm{ppm}\right)$ 2.333 (s, 3H, $\mathrm{CH}_{3}$ ), 1.610-1.243 (d, 2H, C $-\mathrm{H}$ Pyrazoline), 3.981-3.938 (t, 1H, $\mathrm{C}_{5}-\mathrm{H}$ Pyrazoline), 5.016 (d, 1H, NH), 6.921-7.867 (7H, Ar-H). MS (EI) $\mathrm{m} / \mathrm{z}: 333\left(\mathrm{M}^{+}\right)$. Anal. Calcd for $\mathrm{C}_{16} \mathrm{H}_{14} \mathrm{BrFN}_{2}: \mathrm{C}, 57.68 ; \mathrm{H}, 4.24$; N, 8.41. Found C, 57.57; H, 4.16; N, 8.35.
5-(3,4-dimethoxyphenyl)-3-(4-fluoro-3-methylphenyl)-I-4,5-dihydro-1Hpyrazole (4f)

Yellowish crystals $(\mathrm{MeOH})$, Yield $=70 \%$; mp $84-86^{\circ} \mathrm{C}$. FT-IR $(\mathrm{KBr})$ $\mathrm{cm}^{-1}$ :1057 (C-F), 2981 (C-CH $\left.\mathrm{CH}_{3} \mathrm{Str}\right), 1531(\mathrm{C}=\mathrm{N}), 1383\left(\mathrm{CH}_{2}\right.$ bend), 3434 $(\mathrm{Ar}-\mathrm{CH}), 1030\left(\mathrm{O}-\mathrm{CH}_{3}\right) .{ }^{1} \mathrm{HNMR}\left(400 \mathrm{MHz}, \mathrm{CDCl}_{3} \delta \mathrm{ppm}\right) 2.244(\mathrm{~s}, 3 \mathrm{H}$, $\left.\mathrm{CH}_{3}\right), 3.944\left(\mathrm{~d}, 6 \mathrm{H}, \mathrm{OCH}_{3}\right), 1.551\left(\mathrm{~d}, 2 \mathrm{H}, \mathrm{C}_{4}-\mathrm{H}\right.$ Pyrazoline), 2.396 (t, 1H, $\mathrm{C}_{5}-\mathrm{H}$ Pyrazoline), $4.964(\mathrm{~d}, 1 \mathrm{H}, \mathrm{NH}), 6.87-7.76(6 \mathrm{H}, \mathrm{Ar}-\mathrm{H})$. MS (EI) m/z: $314\left(\mathrm{M}^{+}\right)$. Anal. Calcd for $\mathrm{C}_{18} \mathrm{H}_{19} \mathrm{FN}_{2} \mathrm{O}_{2}: \mathrm{C}, 68.77 ; \mathrm{H}, 6.09$; N, 8.91. Found: C, 68.72; H, 5.99; N, 8.84

\section{5-(4-chlorophenyl)-3-(4-fluoro-3-methylphenyl)-4,5-dihydro- $1 \mathrm{H}$ -} pyrazole $(4 \mathrm{~g})$

Orange crystals $(\mathrm{MeOH})$, Yield $=68 \% ; \mathrm{mp} 100-102^{\circ} \mathrm{C}$. FT-IR $(\mathrm{KBr}) \mathrm{cm}^{-1}$ : 1070 (C-F), 2924 (C-CH ${ }_{3}$ Str), $1542(\mathrm{C}=\mathrm{N}), 1436\left(\mathrm{CH}_{2}\right.$ bend $), 3361(\mathrm{Ar}-\mathrm{CH})$. ${ }^{1} \mathrm{HNMR}\left(400 \mathrm{MHz}, \mathrm{CDCl}_{3} \delta \mathrm{ppm}\right) 2.905\left(\mathrm{~s}, 3 \mathrm{H}, \mathrm{CH}_{3}\right), 2.44-2.680(\mathrm{~d}, 2 \mathrm{H}$, $\mathrm{C}_{4}-\mathrm{H}$ Pyrazoline), 4.41-4.52 (t, $1 \mathrm{H}, \mathrm{C}_{5}-\mathrm{H}$ Pyrazoline), 5.121 (d, $\left.1 \mathrm{H}, \mathrm{NH}\right)$, 7.31-8.01 (7H, Ar-H). MS (EI) m/z: $288\left(\mathrm{M}^{+}\right), 290\left(\mathrm{M}^{+2}\right)$ : Anal. Calcd for $\mathrm{C}_{16} \mathrm{H}_{14} \mathrm{ClFN}_{2}: \mathrm{C}, 66.55 ; \mathrm{H}, 4.89 ; \mathrm{N}, 9.70$. Found: C, 66.52; H, 4.81; N, 9.61 4-(3-(4-fluoro-3-methylphenyl)-4,5-dihydro-1 H-pyrazol-5-yl)-N,N-dimethylaniline (4h)

Orange red crystals $(\mathrm{MeOH})$, Yield $=72 \%$; mp $89-91^{\circ} \mathrm{C}$, FT-IR $(\mathrm{KBr})$ $\mathrm{cm}^{-1}: 1024(\mathrm{C}-\mathrm{F}), 2966$ and $1357\left(\mathrm{C}-\mathrm{CH}_{3}\right.$ Str), $1684(\mathrm{C}=\mathrm{N}), 1609$ and 1472 $(\mathrm{C}=\mathrm{C}), 1392\left(\mathrm{CH}_{2}\right.$ bend $), 3060(\mathrm{Ar}-\mathrm{CH}) .{ }^{1} \mathrm{HNMR}\left(400 \mathrm{MHz}, \mathrm{CDCl}_{3}\right.$, $\delta$ ppm) $2.38\left(\mathrm{~s}, 3 \mathrm{H}, \mathrm{CH}_{3}\right), 3.15\left(\mathrm{~s}, 6 \mathrm{H}, \mathrm{N}-\mathrm{CH}_{3}\right), 1.70-1.85\left(\mathrm{~d}, 2 \mathrm{H}, \mathrm{C}_{4}-\mathrm{H}\right.$ Pyrazoline), 3.61-3.84 (t, $1 \mathrm{H}, \mathrm{C}_{5}-\mathrm{H}$ Pyrazoline), 4.861 (d, $\left.1 \mathrm{H}, \mathrm{NH}\right), 6.71$ $7.72(\mathrm{~m}, 7 \mathrm{H}, \mathrm{Ar}-\mathrm{H})$. MS (EI) m/z: $297\left(\mathrm{M}^{+}\right)$. Anal. Calcd for $\mathrm{C}_{18} \mathrm{H}_{20} \mathrm{FN}_{3}$ : C, 72.70; H, 6.78; F, 6.39; N, 14.13. Found C, 72.64; H, 6.28; N, 14.06.

4-(3-(4-fluoro-3-methylphenyl)-4,5-dihydro-1H-pyrazol-5-yl)-2methoxy-6-nitrophenol (4i)

Orange crystals $(\mathrm{MeOH})$, Yield $=70 \%$; mp 93-95० $\mathrm{C}$. FT-IR $(\mathrm{KBr}) \mathrm{cm}^{-1}$ : 1108 (C-F), 2921 and 1299 (C-CH $\mathrm{CH}_{3}$ Str), $1651(\mathrm{C}=\mathrm{N}), 1374\left(\mathrm{CH}_{2}\right.$ bend), $1017\left(\mathrm{OCH}_{3}\right), 3116(\mathrm{Ar}-\mathrm{CH}), 3317(\mathrm{OH}), 1554$ and $1374\left(\mathrm{C}-\mathrm{NO}_{2}, \mathrm{Str}\right)$. ${ }^{1} \mathrm{HNMR}\left(400 \mathrm{MHz}, \mathrm{CDCl}_{3} \delta \mathrm{ppm}\right) 2.365\left(\mathrm{~s}, 3 \mathrm{H}, \mathrm{CH}_{3}\right), 1.684-1.80(\mathrm{~d}, 2 \mathrm{H}$, $\mathrm{C}_{4}$-H Pyrazoline), 4.42-4.58 (t, $1 \mathrm{H}, \mathrm{C}_{5}-\mathrm{H}$ Pyrazoline), 3.39(s, 3H, $\mathrm{OCH}_{3}$ ), 5.27 (s, 1H, NH), 7.19-7.91 (5H, Ar-H). MS (EI) m/z: $346\left(\mathrm{M}^{+}\right)$. Anal. Calcd for $\mathrm{C}_{17} \mathrm{H}_{16} \mathrm{FN}_{3} \mathrm{O}_{4}$ : C, 59.13; H, 4.67; N, 12.17. Found: C, 59.03; H, 4.56; N, 12.09

3-bromo-2-(3-(4-fluoro-3-methylphenyl)-4,5-dihydro-1H-pyrazol-5-yl)6-methoxyphenol (4j)

Greenish crystals $(\mathrm{MeOH})$, Yield $=70 \%$; mp $102-104^{\circ} \mathrm{C}$. FT-IR $(\mathrm{KBr})$ $\mathrm{cm}^{-1}$ :991 (C-F), $2882\left(\mathrm{C}-\mathrm{CH}_{3} \mathrm{Str}\right), 1512(\mathrm{C}=\mathrm{N}), 1464\left(\mathrm{CH}_{2}\right.$ bend $), 3212$ (Ar-CH). ${ }^{1} \mathrm{HNMR}\left(400 \mathrm{MHz}, \mathrm{CDCl}_{3} \delta \mathrm{ppm}\right) 2.09$ (s, 3H, $\left.\mathrm{CH}_{3}\right), 1.41-1.90$ (d, 2H, C $-\mathrm{H}$ Pyrazoline), 4.55-4.71 ( $\mathrm{t}, 1 \mathrm{H}, \mathrm{C}_{5}-\mathrm{H}$ Pyrazoline), 3.412 (s, 3H, O-CH $), 5.102(\mathrm{~d}, 1 \mathrm{H}, \mathrm{NH}), 5.87(\mathrm{~s}, 1 \mathrm{H}, \mathrm{OH}), 6.84-7.94(5 \mathrm{H}, \mathrm{Ar}-\mathrm{H})$. MS (EI) m/z: $378\left(\mathrm{M}^{+}\right)$. Anal. Calcd for $\mathrm{C}_{17} \mathrm{H}_{16} \mathrm{BrFN}_{2} \mathrm{O}_{2}: \mathrm{C}, 53.84 ; \mathrm{H}$, 4.25; N, 7.39; Found: C, 53.76; H, 4.18; N, 7.31

Preparation of 2-(benzo [d] oxazol-2-ylthio) - 1 - (3(4-fluoro-3-methyl phenyl) -5-(substituted phenyl)-4,5dihydro-1H-pyrazol-1-yl)ethanone (6a-6j)

3,5-Diaryl-2-pyrazoline (4a-4j) (2mmol) and triethylamine (2mmol) were added to acetone $(30 \mathrm{~mL})$ and dissolved with constant stirring. Later, the mixture was cooled in an ice bath and chloroacetylchloride $(0.02 \mathrm{~mol})$ was added drop wise with constant stirring. The reaction mixture thus obtained was further agitated for $1 \mathrm{~h}$ at room temperature. Benzo[d]oxazole-2-thiol $(0.01 \mathrm{~mol})$ and $\mathrm{K}_{2} \mathrm{CO}_{3}(0.01 \mathrm{~mol})$ was added to the reaction mixture and refluxed for $8 \mathrm{~h} .{ }^{23}$ The reaction was monitored using TLC. After completion of the reaction, the reaction mixture was cooled and the solution was evaporated until dryness. The residue was washed with water and recrystallized from ethanol. 
Venugopal, et al.: 2-Benzo [d]oxazol-2ylthio Linked Pyrazolines as Potent Anti-TB Agents

2-(benzo[d]oxazol-2-ylthio)-1-(3-(4-fluoro-3-methylphenyl)-5(thiophen-2-yl)-4,5-dihydro-1H-pyrazol-1-yl)ethanone (6a)

Yield $=76$ \%, m.p. $261-263^{\circ} \mathrm{C}$. IR (KBr) cm ${ }^{-1}: 3029(\mathrm{Ar}-\mathrm{CH}), 2970$ $\left(\mathrm{CH}_{3}-\mathrm{CH}\right), 1732(\mathrm{C}=\mathrm{O}), 1639(\mathrm{C}=\mathrm{N}), 1608(\mathrm{C}=\mathrm{C}), 1254(\mathrm{C}-\mathrm{F})$ and 1026 (C-O-C). ${ }^{1} \mathrm{H}-\mathrm{NMR}\left(\mathrm{CDCl}_{3}, 400 \mathrm{MHz}\right) \delta \mathrm{ppm}: 7.06-8.12(\mathrm{~m}, 7 \mathrm{H}, \mathrm{Ar}-\mathrm{CH})$, 6.38-6.60 (m, $3 \mathrm{H}, \mathrm{CH}$ of thiazole), 5.10-5.19 (t, $1 \mathrm{H}, \mathrm{C}_{5}-\mathrm{H}$ of pyrazole), 4.85 (s, 2H, SCH $\mathrm{CO}), 2.93\left(\mathrm{~s}, 3 \mathrm{H}, \mathrm{CH}_{3}\right), 1.98-2.07$ (d, $2 \mathrm{H}, \mathrm{C}_{4}-\mathrm{H}$ of pyrazole). EI-MS $m / z$ : $451\left(\mathrm{M}^{+}\right)$. Anal. Calcd for $\mathrm{C}_{23} \mathrm{H}_{18} \mathrm{FN}_{3} \mathrm{O}_{2} \mathrm{~S}_{2}$ : C, 61.18; H, 4.02; N, 9.31. Found: C, 61.07; H, 3.95; N, 9.12.

2-(benzo[d]oxazol-2-ylthio)-1-(3-(4-fluoro-3-methylphenyl)-5-(3,4,5trimethoxyphenyl)-4,5-dihydro-1H-pyrazol-1-yl)ethanone (6b)

Yield $=74$ \%, m.p. 244-246 ${ }^{\circ} \mathrm{C}$. IR (KBr) cm ${ }^{-1}$ : 3058 (Ar-CH), 2963 $\left(\mathrm{CH}_{3}-\mathrm{CH}\right), 1710(\mathrm{C}=\mathrm{O}), 1648(\mathrm{C}=\mathrm{N}), 1612(\mathrm{C}=\mathrm{C}), 1223(\mathrm{C}-\mathrm{F})$ and 1032 (C-O-C). ${ }^{1} \mathrm{H}-\mathrm{NMR}\left(\mathrm{CDCl}_{3}, 400 \mathrm{MHz}\right) \delta$ ppm: 7.09-8.04 (m, 9H, Ar-CH), 4.98-5.11 (t, $1 \mathrm{H}, \mathrm{C}_{5}-\mathrm{H}$ of pyrazole), 4.36 (s, $\left.2 \mathrm{H}, \mathrm{SCH}_{2} \mathrm{CO}\right), 3.91(\mathrm{~s}, 3 \mathrm{H}$, $\left.\mathrm{OCH}_{3}\right), 3.65$ (s, $\left.3 \mathrm{H}, \mathrm{OCH}_{3}\right), 3.38\left(\mathrm{~s}, 3 \mathrm{H}, \mathrm{OCH}_{3}\right), 2.12\left(\mathrm{~s}, 3 \mathrm{H}, \mathrm{CH}_{3}\right), 1.58-1.80$ (d, $2 \mathrm{H}, \mathrm{C}_{4}-\mathrm{H}$ of pyrazole). EI-MS $m / z: 535\left(\mathrm{M}^{+}\right)$. Anal. Calcd for $\mathrm{C}_{28} \mathrm{H}_{26} \mathrm{FN}_{3} \mathrm{O}_{5} \mathrm{~S}: \mathrm{C}, 62.79 ; \mathrm{H}, 4.89 ; \mathrm{N}, 7.85$ Found: $\mathrm{C}, 62.68 ; \mathrm{H}, 4.75 ; \mathrm{N}$, 7.75 .

2-(benzo[d]oxazol-2-ylthio)-1-(5-(2-bromophenyl)-3-(4-fluoro-3methylphenyl)-4,5-dihydro-1H-pyrazol-1-yl)ethanone (6c)

Yield $=72 \%$, m.p. $234-236^{\circ} \mathrm{C}$. IR $(\mathrm{KBr}) \mathrm{cm}^{-1}: 3095(\mathrm{Ar}-\mathrm{CH}), 2961\left(\mathrm{CH}_{3}-\right.$ $\mathrm{CH}), 1721(\mathrm{C}=\mathrm{O}), 1649(\mathrm{C}=\mathrm{N}), 1623(\mathrm{C}=\mathrm{C}), 1229(\mathrm{C}-\mathrm{F}), 1013(\mathrm{C}-\mathrm{O}-\mathrm{C})$ and $623(\mathrm{C}-\mathrm{Br}) .{ }^{1} \mathrm{H}-\mathrm{NMR}\left(\mathrm{CDCl}_{3}, 400 \mathrm{MHz}\right) \delta \mathrm{ppm}: 7.09-8.05(\mathrm{~m}, 11 \mathrm{H}$, Ar- $\mathrm{CH}$ ), 4.68-4.94 (t, $1 \mathrm{H}, \mathrm{C}_{5}-\mathrm{H}$ of pyrazole), 4.73 (s, 2H, $\left.\mathrm{SCH}_{2} \mathrm{CO}\right), 2.52$ (s, 3H, $\left.\mathrm{CH}_{3}\right), 1.69-1.94\left(\mathrm{~d}, 2 \mathrm{H}, \mathrm{C}_{4}-\mathrm{H}\right.$ of pyrazole). EI-MS $m / z: 525\left(\mathrm{M}^{+2}\right)$, $523\left(\mathrm{M}^{+}\right)$. Anal. Calcd for $\mathrm{C}_{25} \mathrm{H}_{19} \mathrm{BrFN}_{3} \mathrm{O}_{2} \mathrm{~S}$ : C, 57.26; H, 3.65; N, 8.01. Found: C, 57.14; H, 3.60; N, 7.93 .

2-(benzo[d]oxazol-2-ylthio)-1-(3-(4-fluoro-3-methylphenyl)-5-(2nitrophenyl)-4,5-dihydro-1H-pyrazol-1-yl)ethanone (6d)

Yield $=78 \%$, m.p. $256-258^{\circ} \mathrm{C}$. IR ( KBr) cm $\mathrm{cm}^{-1}: 3039$ (Ar-CH), 2937( $\left.\mathrm{CH}_{3}-\mathrm{CH}\right)$, $1757(\mathrm{C}=\mathrm{O}), 1642(\mathrm{C}=\mathrm{N}), 1619(\mathrm{C}=\mathrm{C}), 1536$ and $1340\left(\mathrm{NO}_{2}\right), 1225(\mathrm{C}-\mathrm{F})$ and 1022 (C-O-C). ${ }^{1} \mathrm{H}-\mathrm{NMR}\left(\mathrm{CDCl}_{3}, 400 \mathrm{MHz}\right) \delta$ ppm: 6.91-7.99 (m, 11H, Ar-CH), 4.67-4.90 (t, 1H, $\mathrm{C}_{5}-\mathrm{H}$ of pyrazole), 3.71 (s, $2 \mathrm{H}, \mathrm{SCH}_{2} \mathrm{CO}$ ), 2.36 (s, 3H, $\left.\mathrm{CH}_{3}\right), 1.68-1.92$ (d, 2H, C $-\mathrm{H}$ of pyrazole). EI-MS m/z: 490 $\left(\mathrm{M}^{+}\right)$. Anal. Calcd for $\mathrm{C}_{25} \mathrm{H}_{19} \mathrm{FN}_{4} \mathrm{O}_{4} \mathrm{~S}$ : C, 61.22; $\mathrm{H}, 3.90 ; \mathrm{N}, 11.42$. Found: C, $61.15 ; \mathrm{H}, 3.81 ; \mathrm{N}, 11.36$.

\section{2-(benzo[d]oxazol-2-ylthio)-1-(5-(4-bromophenyl)-3-(4-fluoro-3-} methylphenyl)-4,5-dihydro-1H-pyrazol-1-yl)ethanone (6e)

Yield $=76 \%$, m.p. $230-232^{\circ} \mathrm{C}$. IR $(\mathrm{KBr}) \mathrm{cm}^{-1}: 3045(\mathrm{Ar}-\mathrm{CH}), 2926\left(\mathrm{CH}_{3}-\mathrm{CH}\right)$, $1738(\mathrm{C}=\mathrm{O}), 1645(\mathrm{C}=\mathrm{N}), 1628(\mathrm{C}=\mathrm{C}), 1154(\mathrm{C}-\mathrm{F}), 1021(\mathrm{C}-\mathrm{O}-\mathrm{C})$ and $684(\mathrm{C}-\mathrm{Br}) .{ }^{1} \mathrm{H}-\mathrm{NMR}\left(\mathrm{CDCl}_{3}, 400 \mathrm{MHz}\right) \delta \mathrm{ppm}: 7.04-8.12(\mathrm{~m}, 11 \mathrm{H}$, Ar- $\mathrm{CH}$ ), 4.68-5.06 (t, 1H, $\mathrm{C}_{5}-\mathrm{H}$ of pyrazole), 4.30 (s, 2H, $\left.\mathrm{SCH}_{2} \mathrm{CO}\right), 2.12$ (s, $\left.3 \mathrm{H}, \mathrm{CH}_{3}\right), 1.57-1.92\left(\mathrm{~d}, 2 \mathrm{H}, \mathrm{C}_{4}-\mathrm{H}\right.$ of pyrazole). EI-MS m/z: $525\left(\mathrm{M}^{+2}\right)$, $523\left(\mathrm{M}^{+}\right)$. Anal. Calcd for $\mathrm{C}_{25} \mathrm{H}_{19} \mathrm{BrFN}_{3} \mathrm{O}_{2} \mathrm{~S}$ : C, 57.26; H, 3.65; N, 8.01. Found: C, 57.16; H, 3.61; N, 7.92.

2-(benzo[d]oxazol-2-ylthio)-1-(5-(3,4-dimethoxyphenyl)-3-(4-fluoro-3methylphenyl)-4,5-dihydro-1H-pyrazol-1-yl)ethanone (6f)

Yield $=70 \%$, m.p. $239-241^{\circ} \mathrm{C}$. IR $(\mathrm{KBr}) \mathrm{cm}^{-1}: 3066(\mathrm{Ar}-\mathrm{CH}), 2915\left(\mathrm{CH}_{3}-\mathrm{CH}\right)$, $1752(\mathrm{C}=\mathrm{O}), 1679(\mathrm{C}=\mathrm{N}), 1619(\mathrm{C}=\mathrm{C}), 1204(\mathrm{C}-\mathrm{F})$ and $1010(\mathrm{C}-\mathrm{O}-\mathrm{C})$. ${ }^{1} \mathrm{H}-\mathrm{NMR}\left(\mathrm{CDCl}_{3}, 400 \mathrm{MHz}\right) \delta \mathrm{ppm}$ : 7.14-8.09 (m, 9H, Ar-CH), 4.86-5.06 (t, $1 \mathrm{H}, \mathrm{C}_{5}-\mathrm{H}$ of pyrazole), $4.58\left(\mathrm{~s}, 2 \mathrm{H}, \mathrm{SCH}_{2} \mathrm{CO}\right), 3.61\left(\mathrm{~s}, 3 \mathrm{H}, \mathrm{OCH}_{3}\right), 3.41$ $\left(\mathrm{s}, 3 \mathrm{H}, \mathrm{OCH}_{3}\right), 2.04\left(\mathrm{~s}, 3 \mathrm{H}, \mathrm{CH}_{3}\right), 1.51-1.86\left(\mathrm{~d}, 2 \mathrm{H}, \mathrm{C}_{4}-\mathrm{H}\right.$ of pyrazole). EI-MS m/z: $505\left(\mathrm{M}^{+}\right)$. Anal. Calcd for $\mathrm{C}_{27} \mathrm{H}_{24} \mathrm{FN}_{3} \mathrm{O}_{4} \mathrm{~S}: \mathrm{C}, 64.14 ; \mathrm{H}, 4.78$; N, 8.31. Found: C, 64.06; H, 4.70; N, 8.21. 2-(benzo[d]oxazol-2-ylthio)-1-(5-(4-chlorophenyl)-3-(4-fluoro-3methylphenyl)-4,5-dihydro-1H-pyrazol-1-yl)ethanone (6g)

Yield $=78$ \%, m.p. 233-235 ${ }^{\circ} \mathrm{C}$. IR ( KBr) cm ${ }^{-1}: 3091(\mathrm{Ar}-\mathrm{CH}), 2946\left(\mathrm{CH}_{3}-\mathrm{CH}\right)$, $1781(\mathrm{C}=\mathrm{O}), 1621(\mathrm{C}=\mathrm{N}), 1608(\mathrm{C}=\mathrm{C}), 1161(\mathrm{C}-\mathrm{F}), 1026(\mathrm{C}-\mathrm{O}-\mathrm{C})$ and $684(\mathrm{C}-\mathrm{Cl}) .{ }^{1} \mathrm{H}-\mathrm{NMR}\left(\mathrm{CDCl}_{3}, 400 \mathrm{MHz}\right) \delta \mathrm{ppm}: 7.04-8.12(\mathrm{~m}, 11 \mathrm{H}$, Ar- $\mathrm{CH}$ ), 4.68-5.06 (t, $1 \mathrm{H}, \mathrm{C}_{5}-\mathrm{H}$ of pyrazole), 4.30 (s, 2H, $\left.\mathrm{SCH}_{2} \mathrm{CO}\right), 2.12$ (s, $\left.3 \mathrm{H}, \mathrm{CH}_{3}\right), 1.57-1.92\left(\mathrm{~d}, 2 \mathrm{H}, \mathrm{C}_{4}-\mathrm{H}\right.$ of pyrazole). EI-MS m/z: $525\left(\mathrm{M}^{+2}\right)$, $523\left(\mathrm{M}^{+}\right)$. Anal. Calcd for $\mathrm{C}_{25} \mathrm{H}_{19} \mathrm{ClFN}_{3} \mathrm{O}_{2} \mathrm{~S}: \mathrm{C}, 62.56 ; \mathrm{H}, 3.99 ; \mathrm{N}, 8.76$. Found: C, $62.45 ; \mathrm{H}, 3.87 ; \mathrm{N}, 8.67$

1-(5-(4-dimethylaminophenyl)-3-(4-fluoro-3-methylphenyl)-4,5dihydropyrazol-1-yl)-2-(benzo[d]oxazol-2-ylthio)ethanone (6h)

Yield $=71 \%$, m.p. $226-228^{\circ} \mathrm{C}$. IR $(\mathrm{KBr}) \mathrm{cm}^{-1}: 3351$ and $3220\left(\mathrm{NH}_{2}\right), 3044$ (Ar-CH), $2934\left(\mathrm{CH}_{3}-\mathrm{CH}\right), 1731(\mathrm{C}=\mathrm{O}), 1664(\mathrm{C}=\mathrm{N}), 1625(\mathrm{C}=\mathrm{C}), 1228$ (C-F) and 1045 (C-O-C). ${ }^{1} \mathrm{H}-\mathrm{NMR}\left(\mathrm{CDCl}_{3}, 400 \mathrm{MHz}\right) \delta \mathrm{ppm}: 6.80-7.51$ (m, $11 \mathrm{H}, \mathrm{Ar}-\mathrm{CH}), 4.99-5.20$ (t, $1 \mathrm{H}, \mathrm{C}_{5}-\mathrm{H}$ of pyrazole), 4.17 (s, $2 \mathrm{H}, \mathrm{NH}_{2}$ ), 3.82 (s, $2 \mathrm{H}, \mathrm{SCH}_{2} \mathrm{CO}$ ), 3.11 (s, 6H, N-CH $), 2.17$ (s, 3H, $\mathrm{CH}_{3}$ ), 1.68 1.85 (d, $2 \mathrm{H}, \mathrm{C}_{4}-\mathrm{H}$ of pyrazole). EI-MS m/z: $460\left(\mathrm{M}^{+}\right)$. Anal. Calcd for $\mathrm{C}_{25} \mathrm{H}_{21} \mathrm{FN}_{4} \mathrm{O}_{2} \mathrm{~S}$ : C, 65.20; H, 4.60; N, 12.17. Found: C, 65.12; H, 4.55; N, 12.10

2-(benzo[d]oxazol-2-ylthio)-1-(3-(4-fluoro-3-methylphenyl)-5-(4hydroxy-3-methoxy-5-nitro phenyl)-4,5-dihydro-1H-pyrazol-1-yl) ethanone (6i)

Yield $=73 \%$, m.p. $233-235^{\circ} \mathrm{C}$. IR $(\mathrm{KBr}) \mathrm{cm}^{-1}: 3467(\mathrm{OH}), 3032(\mathrm{Ar}-\mathrm{CH})$, $2952\left(\mathrm{CH}_{3}-\mathrm{CH}\right), 1739(\mathrm{C}=\mathrm{O}), 1636(\mathrm{C}=\mathrm{N}), 1627(\mathrm{C}=\mathrm{C}), 1545$ and 1352 $\left(\mathrm{NO}_{2}\right), 1234(\mathrm{C}-\mathrm{F})$ and 1031 (C-O-C). ${ }^{1} \mathrm{H}-\mathrm{NMR}\left(\mathrm{CDCl}_{3}, 400 \mathrm{MHz}\right) \delta$ ppm: 7.23-8.19 (m, 9H, Ar-CH), $5.46(\mathrm{~s}, 1 \mathrm{H}, \mathrm{OH}), 4.82-5.11\left(\mathrm{t}, 1 \mathrm{H}, \mathrm{C}_{5}-\mathrm{H}\right.$ of pyrazole), 4.09 (s, $\left.2 \mathrm{H}, \mathrm{SCH}_{2} \mathrm{CO}\right), 3.50\left(\mathrm{~s}, 3 \mathrm{H}, \mathrm{OCH}_{3}\right), 2.52(\mathrm{~s}, 3 \mathrm{H}$, $\left.\mathrm{CH}_{3}\right), 1.89-2.04$ (d, 2H, C $-\mathrm{H}$ of pyrazole). EI-MS m/z: $536\left(\mathrm{M}^{+}\right)$. Anal. Calcd for $\mathrm{C}_{26} \mathrm{H}_{21} \mathrm{FN}_{4} \mathrm{O}_{6} \mathrm{~S}$ : C, 58.20; H, 3.95; N, 10.44. Found: C, 58.11; H, $3.85 ; \mathrm{N}, 10.36$.

2-(benzo[d]oxazol-2-ylthio)-1-(5-(6-bromo-2-hydroxy-3-

methoxyphenyl)-3-(4-fluoro-3-methyl phenyl)-4,5-dihydro-1 H-pyrazol1-yl)ethanone (6j)

Yield $=77 \%$, m.p. $263-265^{\circ} \mathrm{C}$. IR $(\mathrm{KBr}) \mathrm{cm}^{-1}: 3429(\mathrm{OH}), 3027(\mathrm{Ar}-\mathrm{CH})$, $2949\left(\mathrm{CH}_{3}-\mathrm{CH}\right), 1715(\mathrm{C}=\mathrm{O}), 1640(\mathrm{C}=\mathrm{N}), 1601$ (C=C), 1242 (C-F) 1027 $(\mathrm{C}-\mathrm{O}-\mathrm{C})$ and $629(\mathrm{C}-\mathrm{Br}) .{ }^{1} \mathrm{H}-\mathrm{NMR}\left(\mathrm{CDCl}_{3}, 400 \mathrm{MHz}\right) \delta \mathrm{ppm}: 7.18-8.35$ (m, 9H, Ar-CH), 5.41 (s, 1H, OH), 4.83-5.06 (t, 1H, $\mathrm{C}_{5}-\mathrm{H}$ of pyrazole), 4.06 (s, 2H, SCH $\mathrm{CO}), 3.49$ (s, 3H, $\left.\mathrm{OCH}_{3}\right), 2.14$ (s, 3H, $\left.\mathrm{CH}_{3}\right), 1.73-1.98$ (d, $2 \mathrm{H}, \mathrm{C}_{4}-\mathrm{H}$ of pyrazole). EI-MS m/z: $571\left(\mathrm{M}^{+2}\right), 569\left(\mathrm{M}^{+}\right)$. Anal. Calcd for $\mathrm{C}_{26} \mathrm{H}_{21} \mathrm{BrFN}_{3} \mathrm{O}_{4} \mathrm{~S}$ : C, 54.74; H, 3.71; N, 7.37. Found: C, 54.57; H, 3.61; N, 7.25

\section{Docking studies}

To know the plausible mode of interactions of the target compounds 6a-6j within the Mycobacterium tuberculosis enoyl reductase enzyme, docking studies ${ }^{24}$ were performed using the X-ray crystal structure of Mycobacterium tuberculosis enoyl reductase (INHA) complexed with reference inhibitor 1-cyclohexyl-n-(3,5-dichlorophenyl)-5oxopyrrolidine-3-carboxamide having resolution $1.62 \AA$ obtained from Protein Data Bank (PDB ID: 4TZK). Docking studies were performed using Autodoc 4.2 software. Preparation of enzymes (Addition of polar hydrogens, addition of $\mathrm{AD} 4$ type atoms, removal of water molecules and heteroatoms) were done with Autodoc 4.2 software as per default settings. Binding site was determined using the previous knowledge of the original ligand's interaction site. For the stimulation runs, each ligand was kept flexible, but the aminoacid residues of active site were kept rigid and default parameters values were taken.

The docking results reveals the energy associated with intermolecular interactions (affinity in $\mathrm{kcal} / \mathrm{mol}$ ) obtained upon computational docking 


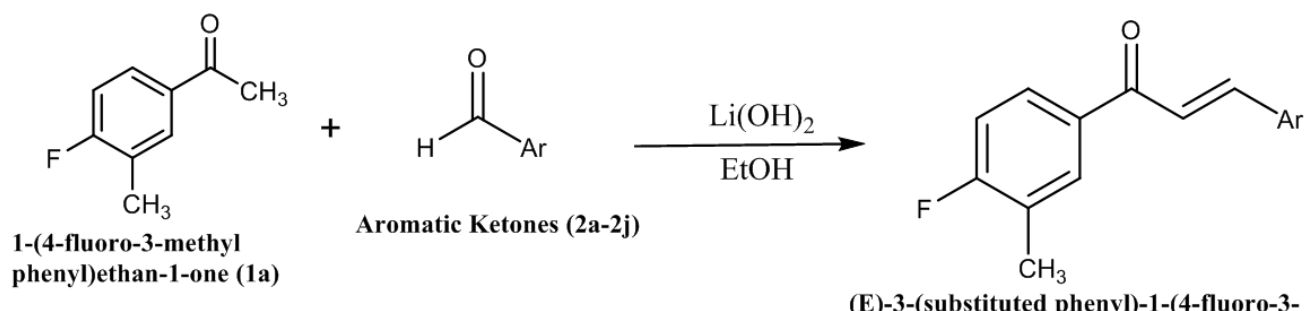
methylphenyl)prop-2-en-1-one (3a-3j)

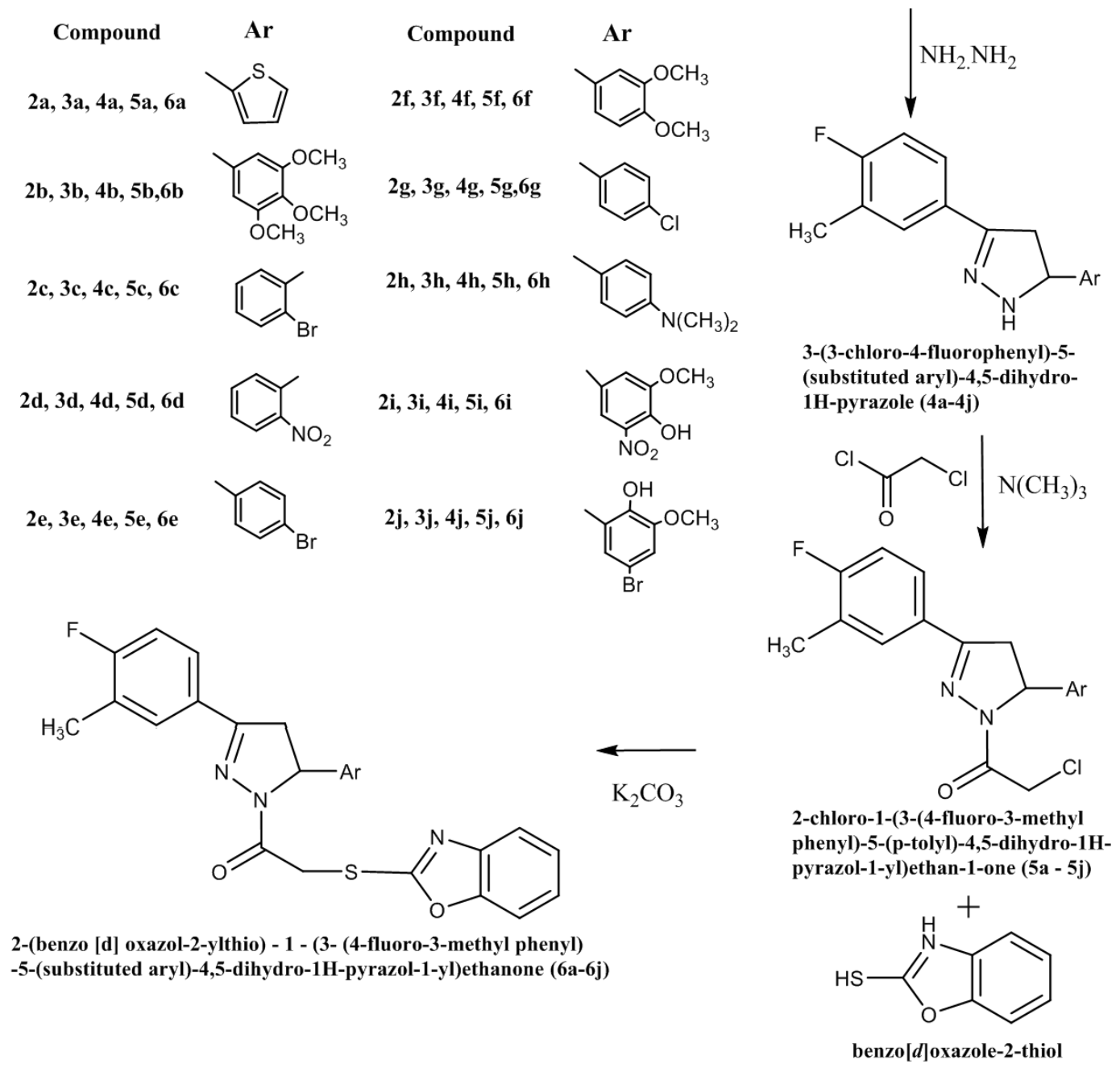

Scheme 1: Synthesis of Pyrazoline derivatives (6a-6j).

for all compounds (6a-6j) within INHA active site and hydrogen bonding interactions between the amino acid residues and functional groups of compounds are summarized in Table 2.

\section{Calculation of pharmacokinetic parameters}

Molinspiration online property calculation toolkit and Osiris property explorer were used to check the pharmaceutical fidelity of the drug candidates. Molecular descriptors, such as number of hydrogen bond donors, the number of hydrogen bond acceptors, the molecular mass of the compounds, topological polar surface area (TPSA), number of rotatable bonds, were calculated using Molinspiration online property calcula-tion toolkit. Percentage of absorption (\%ABS) was calculated by: $\%$ ABS $=109-[0.345 \times$ TPSA $]$

\section{Calculation of toxicity potential}

Osiris Property Explorer was used to analyze various attributes of the drugs, such as toxicity, drug-likeness, and drug score.

\section{Biological Activity}

\section{Anti-tubercular activity}

Anti-tubercular potency of title compounds were estimated by agar dilution method (In vitro M. tuberculosis method). ${ }^{25,26}$ Using OADC growth supplement in Middle brook 7H11 agar slants each test analogs were incorporated in 10 fold serial dilutions. M. tuberculosis $\mathrm{H}_{37} \mathrm{RV}$ inoculums were prepared using OADC growth supplement in fresh Middle brook $7 \mathrm{H} 11$ agar slants adjusted to $1 \mathrm{mg} / \mathrm{ml}$ in $0.05 \%$ tween 80 saline diluted to $10^{-2}\left(10^{7} \mathrm{cfu} / \mathrm{ml}\right.$ concentration approximately). In $7 \mathrm{H} 11$ agar tubes per $\mathrm{ml} 10$ fold serial dilutions of test analogs $5 \mu \mathrm{l}$ 
bacterial suspension was added. At $37^{\circ} \mathrm{C}$ incubated the tubes and after 28 days final readings were measured. Results obtained on test tubes (Test analog, medium and $\mathrm{H}_{37} \mathrm{RV}$ ) were compared with control tubes (Medium and $\mathrm{H}_{37} \mathrm{RV}$ ). The concentration at which complete inhibition of M. tuberculosis growth occurs is known as MIC (minimum inhibitory concentration). INH (Isoniazid), Rifampicin and Ethambutol was used as standard drug for comparing MIC of the title analogs and the obtained results are depicted in Table 1.

\section{RESULTS AND DISCUSSION}

\section{Chemistry}

The protocol for the synthesis of target compounds $6 \mathrm{a}-6 \mathrm{j}$ was shown in Scheme 1 . In this study, a series of novel pyrazoline derivatives $6 \mathrm{a}-6 \mathrm{j}$ were synthesized by substituting various aromatic rings at 5-position

Table 1: MIC (Minimum inhibitory concentration in $\mu \mathrm{g} / \mathrm{ml}$ ) of synthesized compounds.

\begin{tabular}{cc}
\hline Compounds & M. tuberculosis \\
\hline 1 & $>125$ \\
2 & 1.95 \\
3 & 62.5 \\
5 & 125 \\
6 & 62.5 \\
7 & 3.9 \\
8 & 31.25 \\
9 & 1.95 \\
10 & 7.81 \\
Isoniazid & 3.9 \\
Rifampicin & 0.12 \\
Ethambutol & 0.12 \\
\hline
\end{tabular}
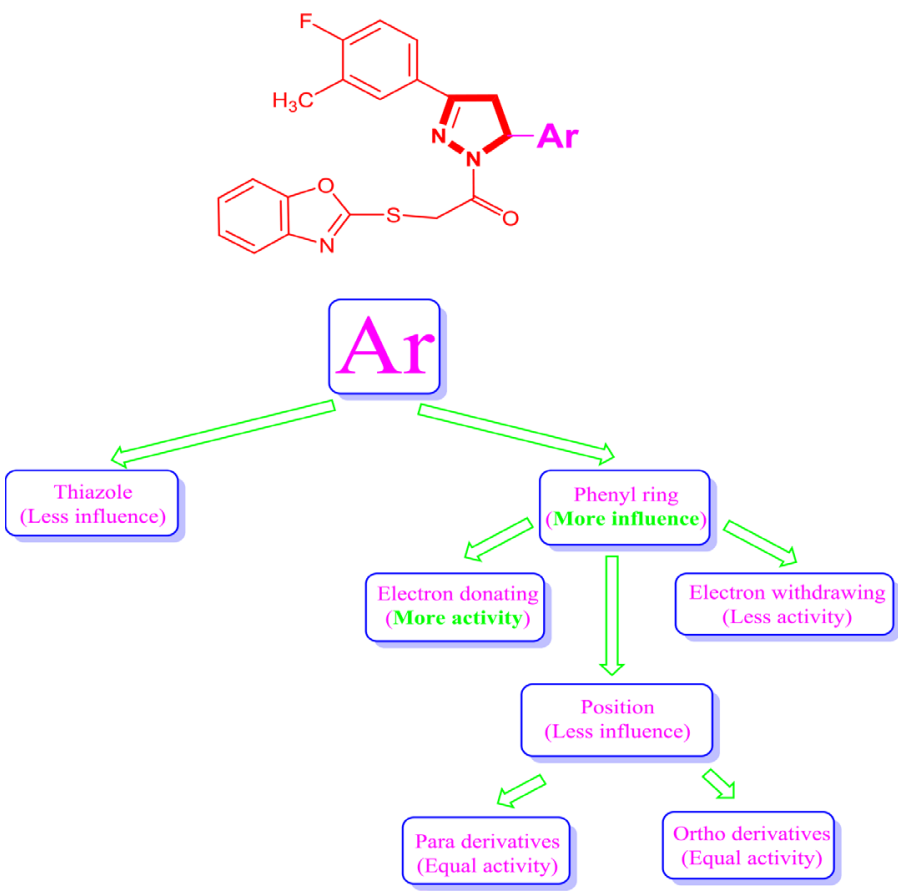

Figure 1: SAR of novel benzoxazole substituted 4,5-dihydropyrazoles (1-10).
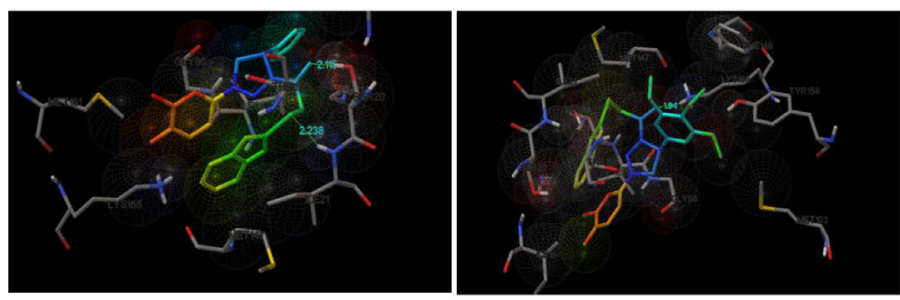

$6 \mathbf{b}$
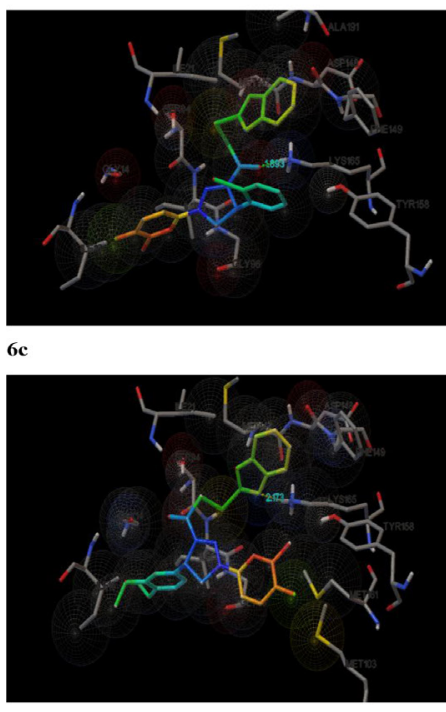

6d

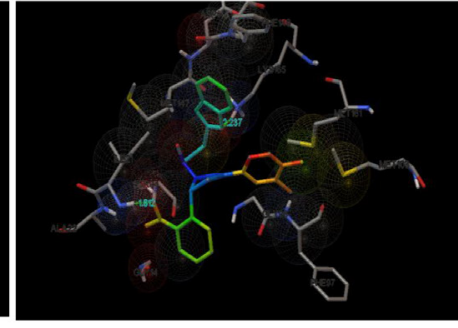

6d
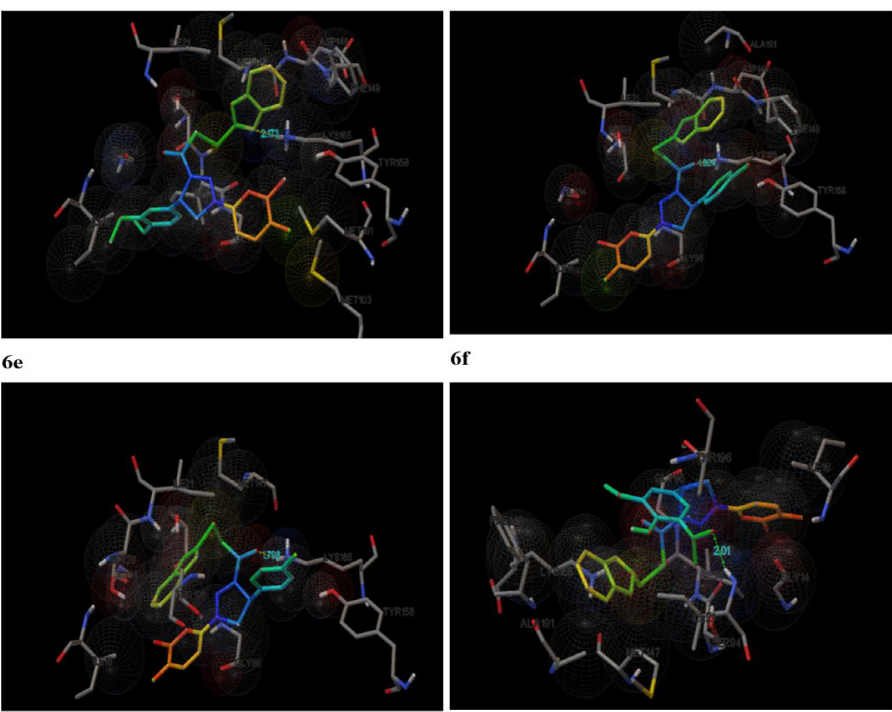

6f

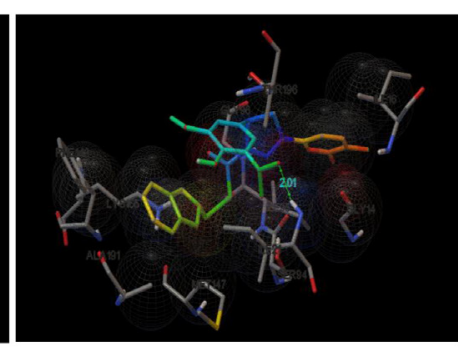

$6 \mathbf{h}$
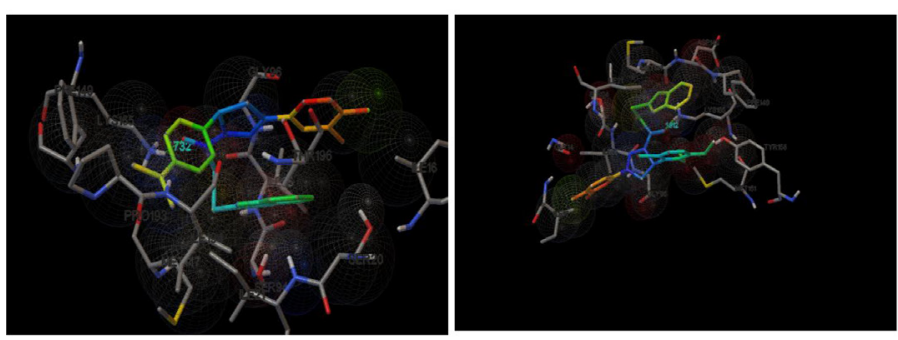

6i

$6 \mathbf{j}$

Figure 2: A view of docked poses between the synthesized compounds and the enzyme.

and 2-benzo[d]oxazol-2-ylthio ethanone moiety at 1-position. By a multistep synthesis, a sequence of new 2-(benzo[d]oxazol-2-ylthio)-1(3-(4-fluoro-3-methylphenyl)-5-(substituted phenyl)-4,5-dihydro-1Hpyrazol-1-yl)ethanone $6 \mathrm{a}-6 \mathrm{j}$ were synthesized from aromatic aldehydes $2 \mathrm{a}-2 \mathrm{j}$ and 4-fluoro-3-methyl acetophenone 1a. Initially 4-fluoro-3methyl acetophenone $1 \mathrm{a}$ was treated with various aromatic aldehydes $2 \mathrm{a}$ $2 \mathrm{j}$ to obtain chalcones $3 \mathrm{a}-3 \mathrm{j}$ by Clasein-Schmidt condensation reaction. Latter, obtained chalcones $3 \mathrm{a}-3 \mathrm{j}$ undergone reaction with hydrazine hydrate in Presence of glacial acetic acid and produced 3-(3-chloro-4fluorophenyl)-5-(substituted phenyl)-4,5-dihydro-1H-pyrazole (4a-4j). 
Table 2: Docking scores of the synthesized compounds.

\begin{tabular}{|c|c|c|c|c|c|}
\hline S.No. & Compound & Docked energy (kcal/mol) & No.of hydrogen bonds & Distance $(\AA \AA)$ from main residue & Aminoacid interactions \\
\hline & \multirow{2}{*}{$6 a$} & \multirow{2}{*}{-8.02} & \multirow{2}{*}{2} & 2.115 & Ser20 \\
\hline & & & & 2.238 & Ile21 \\
\hline & \multirow{2}{*}{$6 \mathrm{~b}$} & \multirow{2}{*}{-9.12} & \multirow{2}{*}{2} & 2.032 & Lys165 \\
\hline & & & & 1.94 & Ser94 \\
\hline & $6 c$ & -8.6 & 1 & 1.893 & Lys165 \\
\hline & \multirow{2}{*}{$6 \mathrm{~d}$} & \multirow{2}{*}{-9.92} & \multirow{2}{*}{2} & 1.812 & ILE21 \\
\hline & & & & 2.237 & Lys165 \\
\hline & $6 e$ & -8.56 & 1 & 1.824 & Ser20 \\
\hline & $6 f$ & -8.99 & 1 & 2.173 & Lys165 \\
\hline & $6 \mathrm{~g}$ & -8.92 & 1 & 1.798 & Ser20 \\
\hline & \multirow{2}{*}{$6 \mathrm{~h}$} & \multirow{2}{*}{-10.48} & \multirow{2}{*}{2} & 2.01 & Ser94 \\
\hline & & & & 2.238 & Ser20 \\
\hline & $6 \mathrm{i}$ & -7.96 & 1 & 2.01 & Ile21 \\
\hline & $6 j$ & -8.25 & 1 & 1.92 & Lys165 \\
\hline
\end{tabular}

Table 3: Drug-likeness/scores and toxicity calculations of ginger compounds based on Osiris property explorer

\begin{tabular}{|c|c|c|c|c|c|c|c|c|c|c|c|}
\hline Compound & TPSA & $\%$ ABS & HBD & HBA & n-ROTB & $\begin{array}{l}\text { Drug- } \\
\text { likeness }\end{array}$ & $\begin{array}{l}\text { Drug } \\
\text { Score }\end{array}$ & Mutagenic & Tumerigenic & Irritant & $\begin{array}{c}\text { Reproductive } \\
\text { effect }\end{array}$ \\
\hline $6 a$ & 112 & 70.36 & 0 & 5 & 5 & 1.39 & 0.51 & green & green & green & green \\
\hline $6 \mathrm{~b}$ & 111.69 & 70.46 & 0 & 8 & 8 & 3.07 & 0.48 & green & green & green & green \\
\hline $6 c$ & 84 & 80.02 & 0 & 5 & 5 & -3.57 & 0.22 & green & green & green & green \\
\hline $6 \mathrm{~d}$ & 129.82 & 64.21 & 0 & 8 & 6 & -7.08 & 0.30 & green & green & green & green \\
\hline $6 e$ & 84 & 80.02 & 0 & 5 & 5 & -0.52 & 0.30 & green & green & green & green \\
\hline $6 g$ & 84 & 80 & 0 & 5 & 5 & 2.43 & 0.46 & green & green & green & green \\
\hline $6 \mathrm{~h}$ & 87.24 & 78.9 & 0 & 6 & 6 & -0.21 & 0.23 & green & red & green & green \\
\hline $6 \mathrm{i}$ & 159.28 & 54.14 & 1 & 10 & 7 & -4.40 & 0.30 & green & green & green & green \\
\hline $6 j$ & 113.46 & 69.85 & 1 & 7 & 6 & -1.35 & 0.25 & green & green & green & green \\
\hline
\end{tabular}

Note: topological polar surface area (TPSA); percentage of absorption (\%ABS); number of hydrogen bond donors (HBD); number of hydrogen bond acceptors (HBA); number of rotatable bonds ( $\mathrm{n}$-ROTB).

In the succeeding step, compounds $(4 \mathrm{a}-4 \mathrm{j})$ were treated with triethyl amine, chloroacetylchloride and benzo[d] oxazole-2-thiol to yield the title compound (6a-6j). Thin-layer chromatography (TLC) was performed throughout the reactions to optimize the reactions for purity and completion.

Infrared (IR), nuclear magnetic resonance (NMR), mass spectra and elemental analyses of the synthesized compounds are in accordance with the assigned structures. The IR spectra of all synthesized compounds showed some characteristic peaks indicating the presence of particular groups. The formations of intermediate chalcones $(2 \mathrm{a}-2 \mathrm{j})$ were confirmed by the presence of IR peak in the region of 1700 to $1600 \mathrm{~cm}^{-1}$ range $(\mathrm{CH}=\mathrm{CH}-\mathrm{C}=\mathrm{O})$. The presence of fluoro group in the chalcones was characterized by the appearance of a strong band in its IR spectrum at $1149 \mathrm{~cm}^{-1}$. The presence of methyl group was confirmed by ${ }^{1} \mathrm{H}$ NMR peak at $\delta 2.3 \mathrm{ppm}$ and IR peak in the region of $2924 \mathrm{~cm}^{-1}$. The IR peak in the region of $1530 \mathrm{~cm}^{-1}$ to $1597 \mathrm{~cm}^{-1}$ range corresponds to $\mathrm{C}=\mathrm{N}$ stretching vibrations with medium intensity indicates the formation of pyrazolines (4a-4j). Its ${ }^{1} \mathrm{H}$ NMR spectrum showed a singlet peak at a range of $\delta 1.226$ $-2.70 \mathrm{ppm}$ due to the proton at the fourth position in the pyrazoline ring confirms its formation. The appearance of IR peak at $1022 \mathrm{~cm}^{-1}$ reveals the attachment of 2-benzo[d]oxazol-2-ylthio ethanone to pyrazoline ring in the final product. The ${ }^{1} \mathrm{H}$ NMR spectrum showed a singlet peak at $\delta 4.09 \mathrm{ppm}$ also supports thio ethanone linkage between pyrazoline and 2-benzo[d]oxazol. The disappearance of ${ }^{1} \mathrm{H}$ NMR peak at 4.96 proved the formation of the title compound (6a-6j). Further mass spectrum confirmed their purity and molecular weight.

\section{Anti-tubercular Activity}

In vitro anti-tubercular activity of all title analogs were screened against M. tuberculosis $\left(\mathrm{H}_{37} \mathrm{Rv}\right.$ strain) and MIC of entire tested analogs were determined and presented in Table 1. Simultaneously MIC of Isoniazid, Rifampicin and Ethambutol was also measured in order to control the sensitivity of the test organisms. From the results it was found that in varying degree synthesized compounds inhibited the growth of $M$. tuberculosis. Among various tested compounds, analogs such as $6 \mathrm{~b}$ and $6 \mathrm{~h}$ inhibited the growth of M. tuberculosis at a low concentration (MIC: $1.95 \mu \mathrm{g} / \mathrm{ml}$ ) which is equal to standard drug Ethambutol. Compound $6 \mathrm{~b}$ possess three methoxy groups and compound $6 \mathrm{~h}$ possess strong 
electron donating dimethylamino moiety at phenyl ring attached to 4,5-dihydropyrazole ring. MIC of test compounds $6 \mathrm{f}$ and $6 \mathrm{j}$ were found to be $3.9 \mu \mathrm{g} / \mathrm{ml}$ may be due to presence of hydroxy and methoxy substituent in phenyl ring. Even though, derivative 6i containing methoxy and hydroxyl moiety completely inhibited the growth of M. tuberculosis only at $7.81 \mu \mathrm{g} / \mathrm{ml}$ concentration because it also contains strong electron withdrawing nitro group. The MIC of analogs $6 \mathrm{~g}$ was found to be $31.25 \mu \mathrm{g} / \mathrm{ml}$ and test compounds $6 \mathrm{c}$ and $6 \mathrm{e}$ were found to be $62.5 \mu \mathrm{g} / \mathrm{ml}$. Only at higher concentration compounds $6 \mathrm{a}$ and $6 \mathrm{~d}$ displayed activity (MIC: $\geq 125 \mu \mathrm{g} / \mathrm{ml}$ ). The MIC of standard drugs isoniazid, Rifamipicin and Ethambutol were found to be $0.12 \mu \mathrm{g} / \mathrm{ml}$, $0.12 \mu \mathrm{g} / \mathrm{ml}$ and $1.95 \mu \mathrm{g} / \mathrm{ml}$, respectively.

\section{Structural Activity Relationship}

SAR of synthesized compounds is depicted in Figure 1. In this study overall it was found that title analogs $6 \mathrm{~b}$ and $6 \mathrm{~h}$ exhibited good antitubercular activity; title analogs $6 \mathrm{f}$ and $6 \mathrm{j}$ showed moderate antitubercular activity; whereas all other title analogs (6a, 6c, 6d, 6e, 6g and 6i) produced only less anti-tubercular activity. In phenyl ring attached to 4,5-dihydropyrazole ring presence of electron donating substituent like dimethylamino, hydroxy and methoxy moiety might be responsible for the powerful anti-bacterial activity displayed by derivatives $6 \mathrm{~b}$ and 6 h. Generally from the SAR study it was found that 4,5-dihydropyrazole derivatives possessing electron releasing group $6 \mathrm{~b}, 6 \mathrm{f}$ and $6 \mathrm{~h}, 6 \mathrm{j}$ exhibited superior anti-tubercular potency than corresponding 4,5-dihydropyrazole derivatives possessing electron withdrawing moieties $6 \mathrm{c}, 6 \mathrm{~d}, 6 \mathrm{e}$ and $6 \mathrm{~g}$. while, thiazole analog 6 a displayed least activity. In addition it was also found that position of the substituent doesn't play any important role in anti-microbial activity because ortho substituted analogs $6 \mathrm{c}$ showed equal activity to corresponding para substituted analogs $6 \mathrm{e}$. Out of ten title compounds, the potent compounds was found to be 2-(benzoxazol2-ylthio)-1-(3-(4-fluoro-3-methylphenyl)-5-(3,4,5-trimethoxyphenyl)4,5-dihydro-1H-pyrazol-1-yl)ethanone 6b and 1-(5-(4-dimethylamino phenyl)-3-(4-fluoro-3-methylphenyl)-4,5-dihydro-1H-pyrazol-1-yl)-2(benzoxazol-2-ylthio) ethanone $6 \mathrm{~h}$.

\section{Docking Studies}

The docking studies, of the title compounds were carried out with Mycobacterium tuberculosis enoyl reductase (INHA) (4TZK.pdb) by using Autodock 4.2 software. The interaction of molecule with Mycobacterium tuberculosis enoyl reductase (INHA) enzyme is shown in Figure 2. The docking results of the target compounds revealed that all the compounds were energetically favorable in terms of Autodock score (Table 2). The Autodock score of the most potent compounds, $6 \mathrm{~b}$ and $6 \mathrm{~h}$ were -9.12 and -10.48 respectively. The dock score of the less active compounds was found more when compared to the active compounds. These results indicate that the more potent compounds require less energy for good binding interaction with the receptor enzyme, whereas the less active compounds require more energy than the high active molecules. The binding interaction of the most active compound, $6 \mathrm{~b}$ showed that the ketone linkage at $\mathrm{N} 1$ of pyrazoline binds with the amino acid residues LYS165 through hydrogen bonding. These binding interactions reveal the importance of the N1 ketone linkage for favorable binding interaction, so that better Mycobacterium tuberculosis enoyl reductase inhibitory activity is expected.

\section{Toxicity risks and drug score assessment}

Nowadays, it is much more convenient to predict the toxicity risks of compounds through reliable bioinformatics tools. In the present study, toxicity risks parameters such as mutagenicity, tumorigenicity, irritation, and reproductive or developmental toxicity of all the synthesized compounds (6a-6j) was calculated by using Osiris property explorer (Table 3). The predictions are based on the functional group similarity for the query derivatives with the in vitro and in vivo validated compounds present in the database of this online program. The toxicity risk predictor locates fragments within a molecule, which indicate a potential toxicity risk. The results can be visualized using color codes; green color shows low tendency of toxicity, yellow shows the mediocre, and red color shows potent tendency of toxicity. However, compound $6 \mathrm{~h}$ indicated high risk of tumerogenicity. In addition, the bioavailability of synthesized compounds was judged through TPSA analysis.

This descriptor has been reported to correlate with passive molecular transport through membranes and therefore, allows prediction of transport properties of drugs and has been linked to drug bioavail $\neg$ ability. Veber's rule states that for good oral bioavailability of a drug, the number of rotatable bond must be $\leq 10$, and TPSA values $\leq 140 \AA 2.27$ The number of rotatable bonds has been shown to be a very good descriptor of oral bioavailability of the drugs. Rotatable bond is defined as any single nonring bond, bounded to non-terminal heavy (ie, non-hydrogen) atom. Amide $\mathrm{C}-\mathrm{N}$ bonds are not considered because of their high rotational energy barrier. Number of rotatable bonds was found to be appropriate in all the compounds. Percentage of absorption was estimated using the equation: \%ABS $=109-0.345 \times$ TPSA, according to Zhao et al. ${ }^{28}$ TPSA was also calculated using Molinspiration online property calculation toolkit according to the fragment-based method of Ertl et al. ${ }^{29}$

\section{CONCLUSION}

In conclusion, we synthesized new 2-pyrazoline derivatives bearing 2-benzo[d]oxazol ring and aromatic substitutions by the reaction of chalcones with hydrazine hydride and 2-benzo[d]oxazol thiol. The structures of obtained compounds were confirmed by spectroscopic methods. All newly obtained pyrazoline derivatives were tested in vitro against Mycobacterium strain: $\mathrm{H}_{37} \mathrm{Rv}$. The most active compopunds against Mycobacterium tuberculosis are compounds $6 \mathrm{~b}$ and $6 \mathrm{~h}$ with trimethoxy phenyl group and 4-dimethylamino phenyl group. The molecular docking studies investigating pyrazoline derivatives using the enzyme Mycobacterium tuberculosis enoyl reductase (INHA) as their potential biological target indicated that the substituted group on pyrazoline spacer play an important role in interactions with the active site of INHA, LYS165, PHE149, ASP148, MET147, TYR158, ILE21 and SER20 as the most active amino acid residues. In the in silico studies, these test compounds showed minimum binding energy with Mycobacterium tuberculosis enoyl reductase (INHA) enzyme. So the present study provides us insight for the further development of better antitubercular agents as Mycobacterium tuberculosis enoyl reductase (INHA) inhibitors

\section{ACKNOWLEDGEMENT}

The authors are thankful to the UGC (New Delhi, India) for providing financial assistance to Department of Pharmacy, GITAM Institute of Pharmacy, Gandhi Institute of Technology and Management (GITAM Deemed to be University), Visakhapatnam, Andhra Pradesh, India.

\section{CONFLICT OF INTEREST}

The authors declare that they have no conflict of interest.

\section{ABBREVIATIONS}

FT:IR: Fourier Transmission Infra Red; ${ }^{1} \mathbf{H}: N M R:$ Proton Nuclear Magnetic resonance; TB: Tuberculosis; XDR: extensively drug:resistant; MDR: Multiple drug:resistant; CNS: Central Nervous System; TLC: Thin Layer chromatography; TPSA: Topical Polar Surface Area; INHA: Mycobacterium tuberculosis enoyl reductase. 


\section{REFERENCES}

1. Lienhardt C, Glaziou P, Uplekar M, Lönnroth K, Getahun H, Raviglione M. Global tuberculosis control: Lessons learnt and future prospects. Nat Rev Microbiol. 2012;10(6):407-16

2. National committee for clinical laboratory standards: Antimycobacterial susceptibility testing for M. tuberculosis. Proposed standards M24-T. Villanova, PA: National committee for clinical laboratory standards. 1995

3. Daniel TM. The history of tuberculosis. Respir Med. 2006:100(11):1862-70.

4. Sriram D, Yogeeswari P, Dinakaran M, Thirumurugan R. Antimycobacterial activity of novel 1-(5-cyclobutyl-1,3-oxazol-2-yl)-3-(substituted)phenyl/pyridyl thiourea endowed with high activity toward multi drug resistant tuberculosis. J Antimicrob Chemother. 2007;59(6):1194-6.

5. Ramesh V, Pardeep K, Bharti M. Revised National Tuberculosis Control Program in India: The Need to Strengthen. Int J Prev Med. 2013;4(1):1-5.

6. Juan CP, Anandi M. Drug Resistance Mechanisms in Mycobacterium tuberculosis Antibiotics. 2014;3(3):317-40.

7. Soliman R, Habib NS, Ashour FA, el-Taiebi M. Synthesis and antimicrobial activity of novel pyrazole, pyrazoline, pyrazolinone and pyrazolidinedione derivatives of benzimidazole. Boll Chim Farm. 2001;140(3):140-8.

8. Hassan MF, Al-Mohammadi MM, Khalid AA, Khalid AK. Synthesis and biological evaluation of fluoropyrazolesulfonylurea and thiourea derivatives as possible antidiabetic agents. J Enzyme Inhib Med Chem. 2016;31(sup1):157-63.

9. Jainey PJ, Bhat IK. Antitumor, Analgesic and Anti-inflammatory Activities of Synthesized Pyrazolines. J Young Pharm. 2012;4(2):82-7.

10. Mui MS, Siew BN, Buss AD, Crasta SC, Kah LG, Sue KL. Synthesis of N-1 acidic functionality affording analogues with enhanced antiviral activity against HIV. Bioorg Med Chem Lett. 2002;12(20):679-99

11. Taher AT, Mostafa SMT, El-Sayed ANR, Hilmy EN. Design, synthesis, modeling studies and biological screening of novel pyrazole derivatives as potential analgesic and anti-inflammatory agents. Bioorg Chem. 2019;89:103-23.

12. Amir M, Kumar $H$, Khan SA. Synthesis and pharmacological evaluation of pyrazoline derivatives as new anti-inflammatory and analgesic agents. Bioorg Med Chem Lett. 2008;18(3):918-22.

13. Kumar G, Tanwar O, Kumar J, Akhter M, Sharma S, Pillai CR, et al. Pyrazolepyrazoline as promising novel antimalarial agents: A mechanistic study. Eur J Med Chem. 2018;149:139-47.

14. Akshay K, Varadaraj B, Rajeev GA, Singla K. Synthesis and evaluation of antioxidant activity of novel 3,5-disubstituted-2-pyrazolines. Bull Fac Pharm. 2013;51(2):167-73

15. Savita U, Avinash CT, Sarvesh P, Shailendra KS. 2-pyrazoline derivatives in neuropharmacology: Synthesis, ADME prediction, molecular docking and in vivo biological evaluation. EXCLI J. 2017;16:628-49.

16. Abdel-Aziz AA, El-Azab AS, Bua S, Nocentini A, Abu El-Enin MA, Alanazi $\mathrm{MM}$, et al. Design, synthesis and carbonic anhydrase inhibition activity of benzenesulfonamide-linked novel pyrazoline derivatives. Bioorg Chem. 2019;87:425-31

17. Peat AJ, Garrido D, Boucheron JA, Schweiker SL, Dickerson SH, Wilson JR, et al. Novel GSK-3 inhibitors with improved cellular activity. Bioorg Med Chem Lett. 2004;14(9):2127-30.

18. Kim DC, Lee YR, Yang BS, Shin KJ, Kim BJ, Chung BY, et al. Synthesis and biological evaluations of pyrazolo[3,4-d]pyrimidines as cyclin-dependent kinase 2 inhibitors. Eur J Med Chem. 2003;38(5):525-32.

19. Schenone S, Bruno O Bondavalli $F$ Ranise A et al. Synthesis of 1-(2-chloro2-phenylethyl)-6- methylthio-1H-pyrazolo[3,4-d]pyrimidines 4-amino substituted and their biological evaluation. European Journal of Medicinal Chemistry. 2004;39(2):153-60.

20. Schenone S, Brullo C, Bruno O, Bondavalli F, Mosti L, et al. Synthesis, biological evaluation and docking studies of 4-amino substituted $1 \mathrm{H}$-pyrazolo[3,4-d] pyrimidines. Eur J Med Chem. 2008;43(12):2665-76

21. Boeck $P$, Falcão CA, Leal PC, Yunes RA, Filho VC, Torres-Santos EC, et al. Synthesis of chalcone analogues with increased antileishmanial activity. Bioorg Med Chem. 2006;14(5):1538-45.

22. Seham YH. Synthesis and biological activity of some new pyrazoline and pyrimidine derivatives. J Braz Chem Soc. 2011;22(7):1286-98.

23. Kaplancikli ZA, Turan-Zitouni G, Ozdemir A, Can OV, Chevallet P. Synthesis and antinociceptive activities of some pyrazoline derivatives. Eur J Med Chem. 2009;44(6):2606-10

24. Lipinski CA, Lombardo F, Dominy BW, Feeney PJ. Experimental and computational approaches to estimate solubility and permeability in drug discovery and development settings. Adv Drug Deliv Rev. 2001;46(1-3):3-26.

25. Lienhardt C, Glaziou P, Uplekar M, Lönnroth K, Getahun H, Raviglione M. Globa tuberculosis control: lessons learnt and future prospects. Nat Rev Microbiol. 2012;10(6):407-16

26. Daniel TM. The history of tuberculosis. Respir Med. 2006;100(11):1862-70.

27. Veber DF, Johnson SR, Cheng HY, Smith BR, Ward KW, Kopple KD. Molecular properties that influence the oral bioavailability of drug candidates. J Med Chem. 2002;45(12):2615-23.

28. Zhao Y, Abraham $\mathrm{MH}$, Le J, et al. Rate-limited steps of human oral absorption and QSAR studies. Pharm Res. 2002;19(10):1446-57.

29. Ertl P, Rohde B, Selzer P. Fast calculation of molecular polar surface area as a sum of fragment-based contributions and its application to the prediction of drug transport properties. J Med Chem. 2000;43(20):3714-17.

Article History: Submission Date : 28-03-2020; Revised Date : 23-04-2020; Acceptance Date : 02-06-2020.

Cite this article: Muralidharan V, Raja S, Asha DC. Synthesis, Characterization, Antitubercular Activity and Docking Studies of 2-(benzo [d]oxazol-2-ylthio)-1-(3-(4fluoro-3-methylphenyl)-5-(substituted aryl)-4,5-dihydro-1H-pyrazol-1-yl)ethanone. J Young Pharm. 2020;12(2)Suppl:s1-s9. 